\title{
Subcontinua of inverse limit spaces of unimodal maps
}

\author{
by \\ Karen B r u cks (Milwaukee, WI) and \\ Henk B r u in (Pasadena, CA)
}

\begin{abstract}
We discuss the inverse limit spaces of unimodal interval maps as topological spaces. Based on the combinatorial properties of the unimodal maps, properties of the subcontinua of the inverse limit spaces are studied. Among other results, we give combinatorial conditions for an inverse limit space to have only arc+ray subcontinua as proper (non-trivial) subcontinua. Also, maps are constructed whose inverse limit spaces have the inverse limit spaces of a prescribed set of periodic unimodal maps as subcontinua.
\end{abstract}

1. Introduction. Inverse limit spaces of one-dimensional maps commonly appear as attractors in dynamical systems. In 1967, R. F. Williams [24] showed that hyperbolic one-dimensional attractors are inverse limits of maps on branched one-manifolds. The full attracting sets for certain maps in the Hénon family are homeomorphic to inverse limits of unimodal maps of an interval [7]. Barge and Diamond [6] show that if a $C^{\infty}$ diffeomorphism of the plane $F$ has a hyperbolic fixed point $p$ with a "same-sided" homoclinic tangency such that the eigenvalues of $D F(p)$ satisfy a non-resonance condition, then any unimodal continuum (inverse limit space where all bonding maps are unimodal maps) appears as a subcontinuum of the closure of the unstable manifold of the fixed point $p$. For other examples see $[1,2,18]$.

Given that such inverse limit spaces commonly appear as attractors, one is interested in the topology of these spaces. In particular, when are such inverse limit spaces homeomorphic as topological spaces? Partial results exist; see [4, 9, 15, 20, 23]. Most recently, Barge and Diamond [4] proved that for transitive Markov maps $f$ and $g$ of an interval $I$, if $(I, f)$ is homeomorphic to $(I, g)$ then the algebraic extensions $\mathbb{Q}(\alpha)=\mathbb{Q}(\beta)$ are equal, where $\alpha$ and

1991 Mathematics Subject Classification: Primary 54F15; Secondary 54H20, 58F03, $58 \mathrm{~F} 12$.

The first author supported in part by a Fulbright grant while visiting the Eötvös Loránd University, Budapest, Hungary.

The second author supported by the Göran Gustafsson Foundation. 
$\beta$ are the spectral radii of the transition matrices for $f$ and $g$ respectively. However, it can be difficult to determine whether $\mathbb{Q}(\alpha)=\mathbb{Q}(\beta)$; for related work see $[14,22]$.

It is unknown whether the inverse limit spaces of two non-conjugate transitive unimodal maps can be homeomorphic. Such inverse limit spaces can, at first glance, locally look like a Cantor set cross an arc. However, this is not the case. To begin, endpoints are very common phenomena and the number of endpoints is a topological invariant. Let $f$ be unimodal with turning point $c$ and core $I$ (a reminder of the definition of "core" is given in the next section). From [8] and [13] follows a first approximation to a classification of these spaces: if $c$ is $n$-periodic, then $(I, f)$ has exactly $n$ endpoints, if $c$ is recurrent but not periodic, then $(I, f)$ has uncountably many endpoints, and if $c$ is not recurrent, then $(I, f)$ has no endpoints (the only exception is the full tent map; here $(0,0, \ldots)$ is indeed an endpoint of $(I, f))$.

In this paper we look at inverse limit spaces $(I, f)$ where the bonding map $f: I \rightarrow I$ is unimodal. We attempt to distinguish such inverse limit spaces via their proper subcontinua. The existence and abundance of nontrivial proper subcontinua is established by Barge et al. [3]. Working with the family $\left\{T_{a}\right\}$ of tent maps ( $a$ is the slope), they exhibit a residual full Lebesgue measure set of slopes such that if $T_{a}$ is a tent map with such a slope, then $\left(I, T_{a}\right)$ is nowhere locally homeomorphic to the product of a Cantor set and an arc. More precisely, every open set in $\left(I, T_{a}\right)$ contains a homeomorphic copy of every inverse limit space $\left(I, T_{a_{i}}\right)$ appearing in this family of inverse limit spaces (the slopes $a_{i}$ are allowed to vary). Hence, there is a form of self-similarity and local recapitulation of the entire family of inverse limits of tent maps within $\left(I, T_{a}\right)$.

In this work, we try to describe which sorts of subcontinua can be found in $(I, f)$ depending on the combinatorial structure of $f$. The simplest subcontinua are points and arcs. In Section 3, for any subcontinuum $H$ of $(I, f)$ which is not simply a point, we construct a dense ray $R_{H}$ within $H$. When $H \backslash R_{H}$ consists of one or two arcs, we call $H$ an arc+ray continuum. The $\sin (1 / x)$-continuum is an example of an arc+ray continuum. In Section 4 we give combinatorial conditions on $f$ under which $(I, f)$ has only arcs or only arc+ray subcontinua. Three examples are given in that section. It is easy to prove that if $f$ is long-branched, then all proper subcontinua of $(I, f)$ are points or arcs (see Sections 2 and 4 ). We provide an example of a map $g$ which is not long-branched, but such that all proper subcontinua of $(I, g)$ are points or arcs. In the second example the $(I, f)$ constructed contains an MW-continuum (a non-homeomorphic variation of the $\sin (1 / x)$-continuum). From this example and the proof of Theorem 1 one can easily construct examples to obtain other non-homeomorphic variations 
of the $\sin (1 / x)$-continuum as subcontinua. Lastly, we completely describe the proper subcontinua of $(I, f)$ when $f$ has the Fibonacci combinatorics.

In Section 5, we apply the framework of kneading maps to obtain subcontinua that are homeomorphic to a given unimodal map with fixed combinatorial structure. This leads to the following result: Let $\mathcal{F}$ be a finite or infinite sequence of maps with periodic turning points. We supply a map $g$ such that for each $f \in \mathcal{F},(I, g)$ contains a subcontinuum $H(f)$ homeomorphic to $(I, f)$. Moreover, every non-arc or non-arc+ray subcontinuum of $(I, g)$ is homeomorphic to $(I, f)$ for some $f \in \mathcal{F}$.

We conjecture that the possible subcontinua are determined by the asymptotic combinatorial structure of the bonding map. To be precise, if the kneading maps of $f$ and $g$ eventually agree, then $(I, f)$ and $(I, g)$ have the same proper subcontinua.

2. Preliminaries. Given continuous maps $f_{i}: I_{i} \rightarrow I_{i-1}$, the associated inverse limit space with bonding maps $f_{i}$ is

$$
\left(I_{i}, f_{i}\right)=\left\{\left(x_{1}, x_{2}, x_{3}, \ldots\right) \mid x_{i-1}=f_{i}\left(x_{i}\right) \text { for } i \geq 2\right\}
$$

we assume that $\left\{\left|I_{i}\right|\right\}_{i}$ is bounded. It has the metric $d(x, y)=\sum_{i}\left|x_{i}-y_{i}\right| / 2^{i}$. The $i$ th projection is denoted by $\pi_{i}:\left(I_{i}, f_{i}\right) \rightarrow I_{i}$. In general, $\left(I_{i}, f_{i}\right)$ is a continuum, i.e. a compact connected metric space. It is one-dimensional and chainable (see [21]). The induced homeomorphism, $\widehat{f}$, of $\left(I_{i}, f_{i}\right)$ is given by $\widehat{f}\left(\left(x_{1}, x_{2}, \ldots\right)\right)=\left(f_{1}\left(x_{1}\right), x_{1}, x_{2}, \ldots\right)$. Set $f_{i, j}=f_{j+1} \circ \ldots \circ f_{i}$.

A continuous map $f:[0,1] \rightarrow[0,1]$ is called unimodal if there exists a unique turning or critical point, $c$, such that $\left.f\right|_{[0, c)}$ is increasing (decreasing) and $\left.f\right|_{(c, 1]}$ is decreasing (increasing). We denote the forward images of $c$ by $c_{i}:=f^{i}(c)$. To avoid trivial cases, we assume that $c$ lies between $c_{1}$ and $c_{2}$. We will discuss the case where there is a single unimodal bonding map $f$. If the map $f:[0,1] \rightarrow[0,1]$ is not onto, then $([0,1], f)=\left(K,\left.f\right|_{K}\right)$ where $K=\bigcap_{n=1}^{\infty} f^{n}([0,1])$. When dealing with a single bonding map $f$ we assume that $f$ is unimodal and that $f(0)=0$ when $f$ has a maximum at $c$ or that $f(1)=1$ when $f$ has a minimum at $c$. Then $\bigcap_{n=1}^{\infty} f^{n}([0,1])$ is either $\left[0, c_{1}\right]$ or $\left[c_{1}, 1\right]$, depending on whether $f$ has a maximum or minimum at $c$. For ease of discussion, assume $f$ has a maximum at $c$. When we write $(I, f)$ we assume that $f:[0,1] \rightarrow[0,1]$ is unimodal with $f(0)=0$ and we set $I \equiv\left[0, c_{1}\right]$. Notice that the interval $\left[c_{2}, c_{1}\right]$ is invariant and $f$ maps $\left[c_{2}, c_{1}\right]$ onto itself; the interval $\left[c_{2}, c_{1}\right]$ is called the core of the map $f$. The inverse limit space $(I, f)$ is identical to $\left(\left[c_{2}, c_{1}\right], f\right)$ except possibly for an additional arc which is an infinite ray entwined with $\left(\left[c_{2}, c_{1}\right], f\right)$.

We further assume that $\left.f\right|_{\left[c_{2}, c_{1}\right]}$ is locally eventually onto (leo), i.e., for every subinterval $J \subset\left[c_{2}, c_{1}\right]$, there exists $N$ such that $f^{n}(J)=\left[c_{2}, c_{1}\right]$ for all $n \geq N$. This condition is not as restrictive as it may first appear, be- 
cause $(I, f)$ is homeomorphic to $(I, g)$ whenever $f$ and $g$ are topologically conjugate. Moreover, any unimodal map without restrictive intervals, periodic attractors, or wandering intervals is topologically conjugate to a tent map with slope $>\sqrt{2}$ (see e.g. [19]), and these tent maps are leo. We say a unimodal map $f$ has a periodic turning point provided $f^{n}(c)=c$ for some $n$.

An $\operatorname{arc}$ is a continuous one-to-one image of the interval $[0,1]$; a ray is a continuous one-to-one image of $(0,1)$ or $(0,1]$. Let $[a, b]$ denote the interval with endpoints $a$ and $b$, irrespective whether $a \leq b$ or $b \leq a$. Set $T_{a}(x)=a x$ for $0 \leq x \leq 1 / 2$ and $T_{a}(x)=a(1-x)$ for $1 / 2 \leq x \leq 1$. We call $T_{2}$ the full tent map. The one-parameter family $\left\{T_{a} \mid a \in[0,2]\right\}$ is referred to as the tent family.

LEMma 1. If $H$ is a subcontinuum of $(I, f)$ then $\pi_{i} H$ is an interval for each $i$. If $\pi_{i} H \ni c$ for only finitely many $i$, then $H$ is an arc or a point.

Proof. As $\pi_{i}$ is continuous for each $i, \pi_{i} H$ is compact and connected. Let $n$ be so large that $c \notin \pi_{i} H$ for $i>n$. Then $H$ can be parametrized by a single variable $t \in \pi_{n} H$.

Lemma 2. A subcontinuum $H \subset\left(\left[c_{2}, c_{1}\right], f\right)$ is proper if and only if $\left|\pi_{i} H\right| \rightarrow 0$ as $i \rightarrow \infty$.

Proof. The if-direction is trivial. Suppose now that there exists $\varepsilon>0$ and a sequence $\left\{m_{i}\right\}$ such that $\left|\pi_{m_{i}} H\right|>\varepsilon$. By passing to a subsequence, we can assume that $\pi_{m_{i}} H \supset J$ for some interval with $|J|>\varepsilon / 2$. Since $f$ is leo, there exists $N$ such that $f^{N}(J)=\left[c_{2}, c_{1}\right]$. It follows that $\pi_{m_{i}-N} H \supset\left[c_{2}, c_{1}\right]$ for all $i$. Hence $H$ is not a proper subcontinuum.

Apart from points and arcs, far more complicated subcontinua may be present. One class pertains to the subcontinua consisting of a ray and one or two arcs. We call such a continuum an arc+ray continuum. This arc+ray continuum is one-sided if it is the closure of the graph of an oscillating function $h:[0,1) \rightarrow[0,1]$. Examples are the $\sin (1 / x)$-continuum and the M-continuum [21, page 41]. The two-sided arc+ray continuum is the closure of the graph of an oscillating function $h:(0,1) \rightarrow[0,1]$ (infinitely many oscillations near both 0 and 1 ).

For $x<c$, let $\widehat{x}>c$ be the point such that $f(x)=f(\widehat{x})$. It will be useful to have a symbolic description of $(I, f)$. For $x \in(I, f)$, let $\nu(x) \in\{0, *, 1\}^{\mathbb{N}}$, called the itinerary of $x$, be defined as

$$
\nu_{j}(x)= \begin{cases}0 & \text { if } \pi_{j}(x)<c \\ * & \text { if } \pi_{j}(x)=c \\ 1 & \text { if } \pi_{j}(x)>c\end{cases}
$$

Let $f^{n}$ be some iterate of $f$ and let $J$ be any maximal subinterval on which $\left.f^{n}\right|_{J}$ is monotone. Then $f^{n}: J \rightarrow I$ is called a branch of $f^{n}$. A map 
is called long-branched if there exists $\varepsilon>0$ such that $\left|f^{n}(J)\right|>\varepsilon$ for every $n$ and every branch $f^{n}: J \rightarrow I$ of $f^{n}$. For example, since $\partial f^{n}(J) \subset$ $\operatorname{orb}(c) \cup\{0\}$, any map with a finite critical orbit is long-branched.

A branch $f^{n}: J \rightarrow I$ is called a central branch if $c \in \partial J$. Hence there are always two central branches, the images of which are the same. An iterate $n$ is called a cutting time if the image of the central branch of $f^{n}$ contains $c$. The subsequent cutting times are denoted by $S_{0}, S_{1}, S_{2}, \ldots\left(S_{0}=1\right)$. If $f^{S_{k}}: J \rightarrow I$ is the left central branch of $f^{S_{k}}$, then there is a unique point $z_{k} \in J$ such that $f^{S_{k}}\left(z_{k}\right)=c$. By construction, $z_{k}$ has the property that $\bigcup_{0<j \leq S_{k}} f^{-j}(c) \cap\left(z_{k}, c\right)=\emptyset$ and is therefore called a closest precritical point. The symmetric point $\widehat{z}_{k}$ is also a closest precritical point. It can be proven that the difference of two subsequent cutting times is again a cutting time. Hence we can write

$$
S_{k}-S_{k-1}=S_{Q(k)},
$$

where $Q: \mathbb{N} \rightarrow \mathbb{N}$ is an integer function, called the kneading map. An equivalent statement is

$$
f^{S_{k}}(c) \in\left(z_{Q(k+1)-1}, z_{Q(k+1)}\right] \cup\left[\widehat{z}_{Q(k+1)}, \widehat{z}_{Q(k+1)-1}\right) .
$$

The kneading map was introduced by Hofbauer (see e.g. [16, 17]). A survey of this tool can be found in [12]. Clearly, every unimodal map has a kneading map. Conversely, an integer map $Q$ is admissible, i.e. is realized as the kneading map of some unimodal map, if the following admissibility condition holds:

$$
\{Q(k+j)\}_{j \geq 1} \succeq\left\{Q\left(Q^{2}(k)+j\right)\right\}_{j \geq 1} \quad \text { for all } k \geq 1 .
$$

Here $\succeq$ denotes the lexicographical ordering. The kneading map (or cutting times) determine the combinatorics of $f$ completely, and therefore the topology of the inverse limit space. Here it is important to have $f$ leo, so that ambiguities involving e.g. stable periodic orbits cannot occur (see [19, Section II.3]).

3. Dense rays. For a subcontinuum $H \subset(I, f)$, let $\left\{n_{i}\right\}_{i \geq 1}$ be the set of critical projections, i.e. the integers $n_{i}$ such that $c \in \operatorname{int} \pi_{n_{i}} H$. By applying $\widehat{f}^{-1}$ to $H$, we may assume that $n_{1}=1$. Only the asymptotic behaviour of the critical projections is important.

Lemma 3. Let $H \subset(I, f)$ be a subcontinuum with critical projections $\left\{n_{i}\right\}$.

(i) For each $i \geq 2$, there exist $k_{i}$ such that $n_{i}-n_{i-1}=S_{k_{i}}$. Moreover, $c_{S_{k_{i}}} \in \partial \pi_{n_{i-1}} H$.

(ii) For each $i \geq 1, j \geq 0$, either $n_{i}-S_{k_{i}+j}$ is a critical projection, or $n_{i}-S_{k_{i}+j}<n_{1}$. 
Proof. For $i \geq 2$, let $k_{i}$ be minimal such that $z_{k_{i}}$ or $\widehat{z}_{k_{i}} \in \pi_{n_{i}} H$. Because $z_{k_{i}}$ is a closest precritical point, $n_{i}-S_{k_{i}}=\max \left\{n<n_{i} \mid \pi_{n} H \ni c\right\}$. Therefore $n_{i}-S_{k_{i}}=n_{i-1}$. Since $c_{1} \in \partial \pi_{n_{i}-1} H$ and $f^{j}\left(\pi_{n_{i}-1} H\right) \not \supset c$ for $j<S_{k_{i}}$, also $c_{S_{k_{i}}} \in \partial \pi_{n_{i-1}} H$. This proves the first statement. We now have $\pi_{n_{i}} H \supset\left[z_{k_{i}}, c\right]$ or $\left[c, \widehat{z}_{k_{i}}\right]$. Therefore also $\pi_{n_{i}} H \ni z_{k_{i}+j}$ or $\widehat{z}_{k_{i}+j}$ for each $j \geq 0$. It follows that $f^{S_{k_{i}+j}}\left(\pi_{n_{i}} H\right) \ni$ c, i.e. $n_{i}-S_{k_{i}+j} \in\left\{n_{m}\right\}$ for each $j \geq 0$ or $n_{i}-S_{k_{i}+j}<n_{1}$.

Definition 1. Let $H \subset(I, f)$ be a subcontinuum with critical projections $\left\{n_{i}\right\}$. For $i \geq 1$ let $M_{n_{i}}$ be the closure of a component of $\pi_{n_{i}} H \backslash\{c\}$ such that

$$
f^{S_{k_{i}}}\left(M_{n_{i}}\right)=\pi_{n_{i-1}} H .
$$

and let $L_{n_{i}}$ be the closure of the remaining component.

In Definition 1, it may be the case that the closure of each component of $\pi_{n_{i}} H \backslash\{c\}$ satisfies (4). In this case, let $L_{n_{i}}$ be the component containing $c_{S_{k_{i+1}}}$.

Proposition 1. Let $H \subset(I, f)$ be a subcontinuum. Then $H$ is a point or contains a dense ray.

Proof. Let $\varphi_{1}:[0,1] \rightarrow \pi_{n_{1}} H$ be linear, onto, and such that $\varphi_{1}(1)=$ $c_{S_{k_{2}}}$. Set $\alpha_{2}=0$ and $\beta_{2}=1$. Recursively define $\varphi_{i}:\left[\alpha_{i+1}, \beta_{i+1}\right] \rightarrow \pi_{n_{i}} H$ to

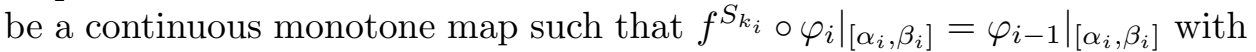
$\varphi_{i}\left(\left[\alpha_{i}, \beta_{i}\right]\right)=M_{n_{i}}$, and such that $\varphi_{i}:\left[\alpha_{i+1}, \beta_{i+1}\right] \backslash\left[\alpha_{i}, \beta_{i}\right] \rightarrow L_{n_{i}}$ is linear and onto. The $\alpha_{i}$ and $\beta_{i}$ are chosen so that $-1<\ldots \leq \alpha_{4} \leq \alpha_{3} \leq \alpha_{2}<$ $\beta_{2} \leq \beta_{3} \leq \beta_{4} \leq \ldots<2$, and so that $\left|\alpha_{i+1}-\alpha_{i}\right|=0$ and $\left|\beta_{i+1}-\beta_{i}\right|=1 / 2^{i}$ or vice versa for all $i$. Let $\alpha=\lim _{i \rightarrow \infty} \alpha_{i}$ and $\beta=\lim _{i \rightarrow \infty} \beta_{i}$. For a fixed $m \geq 1$ and $x \in(\alpha, \beta)$, set $i_{0}=\min \left\{i \mid x \in\left[\alpha_{i+1}, \beta_{i+1}\right]\right.$ and $\left.m \leq n_{i}\right\}$. Then, by construction, $f^{n_{i}-m} \circ \varphi_{i}(x)=f^{n_{i_{0}}-m} \circ \varphi_{i_{0}}(x)$ for $i \geq i_{0}$. Hence, we can take limits and define $\Phi:(\alpha, \beta) \rightarrow H$ as

$$
\Phi_{m}=\lim _{i \rightarrow \infty} f^{n_{i}-m} \circ \varphi_{i} .
$$

As $\Phi$ is one-to-one and continuous, $R=\Phi((\alpha, \beta))$ is a ray. By construction, $\pi_{n} R=\pi_{n} H$ for each $n$. Hence, $R$ lies dense in $H$.

For a map $f$ and integers $\left\{n_{i}\right\}$, the next proposition gives combinatorial conditions which allow a subcontinuum $H \subset(I, f)$ with critical projections precisely $\left\{n_{i}\right\}$. In fact, $H$ is the largest subcontinuum of $(I, f)$ with this set of critical projections, i.e., if $H^{\prime}$ is another subcontinuum, then $H^{\prime} \subset H$ if and only if the critical projections of $H^{\prime}$ are a subset of the critical projections of $H$. 
Proposition 2. Let $f$ and a set of integers $\left\{n_{i}\right\}_{i \geq 1}$ satisfy:

(i) For each $i \geq 2$, there exists $k_{i}$ such that $n_{i}-n_{i-1}=S_{k_{i}}$ with $n_{1}=1$.

(ii) For each such $k_{i}, Q\left(Q\left(k_{i}\right)+1\right)<k_{i-1}$.

(iii) For each $i \geq 2,\left\{S_{k_{i}+j}\right\}_{j \geq 0} \succeq\left\{n_{i}-n_{i-j-1}\right\}_{j \geq 0}$, where $\succeq$ denotes the lexicographical ordering.

Then there is a subcontinuum $H \subset(I, f)$ whose critical projections are precisely $\left\{n_{i}\right\}_{i \geq 1}$. If additionally $k_{i} \rightarrow \infty$, then $H$ is a proper subcontinuum.

Proof. We construct the subcontinuum $H$ by constructing the projections $\pi_{n} H$. For $n<n_{2}$ let

$$
\pi_{n} H=f^{n_{2}-n}\left(\left[z_{k_{2}-1}, c\right]\right) .
$$

Because $\left[z_{k_{2}-1}, c\right]$ is a maximal interval on which $f^{S_{k_{2}}}$ is monotone, $\pi_{n} H \not \supset$ $c$ for $n_{1}<n<n_{2}$, but $\pi_{n_{1}} H=f^{S_{k_{2}}}\left(\left[z_{k_{2}-1}, c\right]\right)=\left[c_{S_{Q\left(k_{2}\right)}}, c_{S_{k_{2}}}\right] \ni c$. Also, $\pi_{n_{2}-1} H \ni c_{1}$. Let $M_{n_{2}}$ be the interval adjacent to $\left[c, c_{S_{k_{3}}}\right]$ such that $f\left(M_{n_{2}}\right)=\pi_{n_{2}-1} H$, and let

$$
\pi_{n_{2}} H=M_{n_{2}} \cup\left[c, c_{S_{k_{3}}}\right] .
$$

Then $\pi_{n_{2}} H \subset\left[z_{k_{2}-1}, \widehat{z}_{k_{2}-1}\right]$. Indeed, $M_{n_{2}}$ is one of $\left[z_{k_{2}-1}, c\right]$ or $\left[c, \widehat{z}_{k_{2}-1}\right]$ since $\left.f^{S_{k_{2}}}\right|_{M_{n}}$ is monotone and $\left[z_{k_{2}-1}, c\right]$ is a monotonicity interval of $f^{S_{k_{2}}}$. Assumption (iii) and Lemma 3(i) imply that $S_{k_{3}+1} \geq n_{3}-n_{1}$ and therefore that $Q\left(k_{3}+1\right) \geq k_{2}$. Hence, $c_{S_{k_{3}}} \in\left[z_{k_{2}-1}, \widehat{z}_{k_{2}-1}\right]$.

Let us continue the construction under the inductive assumption that

$$
\pi_{n_{i}} H \subset\left[z_{k_{i}-1}, \widehat{z}_{k_{i}-1}\right] \text { and } c_{S_{k_{i+1}}} \in \partial \pi_{n_{i}} H .
$$

This holds, as we just checked, for $i=2$. For $n_{i-1}<n<n_{i}$ let

$$
\pi_{n} H=f^{n_{i}-n}\left(M_{n_{i}}\right)
$$

where $M_{n_{i}}$ is the maximal interval adjacent to $\left[c, c_{S_{k_{i+1}}}\right]$ such that $f^{S_{k_{i}}}\left(M_{n_{i}}\right)$ $=\pi_{n_{i-1}} H$. Now $f^{S_{k_{i}}}$ maps $\left[z_{k_{i}-1}, c\right]$ monotonically on $\left[c_{S_{Q\left(k_{i}\right)}}, c_{S_{k_{i}}}\right]$, and because $Q\left(Q\left(k_{i}\right)+1\right)<k_{i-1}$, we have $\left[c_{S_{Q\left(k_{i}\right)}}, c_{S_{k_{i}}}\right] \supset\left[z_{k_{i-1}-1}, c_{S_{k_{i}}}\right]$. Therefore $M_{n_{i}}$ is well defined and contained in $\left[z_{k_{i}-1}, \widehat{z}_{k_{i}-1}\right]$. Let

$$
\pi_{n_{i}} H=M_{n_{i}} \cup\left[c, c_{S_{k_{i+1}}}\right] .
$$

Again (use assumption (iii) and Lemma 3(i)), $S_{k_{i+1}+1} \geq n_{i+1}-n_{i-1}$ implies $Q\left(k_{i+1}+1\right) \geq k_{i}$, and hence $c_{S_{k_{i+1}}} \in\left[z_{k_{i}-1}, \widehat{z}_{k_{i}-1}\right]$. This proves the induction hypothesis (5) for $i+1$, and we can continue the construction.

In this way we obtain a sequence of compact projections $\pi_{n} H$ with the property that $f\left(\pi_{n} H\right) \supset \pi_{n-1} H$. It remains to show that $f\left(\pi_{n} H\right) \subset \pi_{n-1} H$.

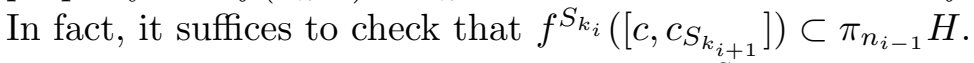

We know that $\left[c, c_{S_{k_{i+1}}}\right] \subset \pi_{n_{i}} H$. Now $f^{S_{k_{i}}}\left(\left[c, c_{S_{k_{i+1}}}\right]\right)=\left[c_{S_{k_{i}}}, c_{S_{k_{i+1}}+S_{k_{i}}}\right]$ is an interval that overlaps $\pi_{n_{i-1}} H$. If $S_{k_{i+1}+1}>n_{i+1}-n_{i-1}=S_{k_{i+1}}+S_{k_{i}}$, then $\left[c_{S_{k_{i}}}, c_{S_{k_{i+1}}+S_{k_{i}}}\right] \not \supset c$. Therefore this interval is contained in $\pi_{n_{i-1}} H$. 
If $S_{k_{i+1}+1}=n_{i+1}-n_{i-1}=S_{k_{i+1}}+S_{k_{i}}$, then $\left[c_{S_{k_{i}}}, c_{S_{k_{i+1}}+S_{k_{i}}}\right] \ni c$. The piece $\left[c, c_{S_{k_{i}}}\right]$ is contained in $\pi_{n_{i-1}} H$. But the piece $\left[c, c_{S_{k_{i+1}+1}}\right]$ is not yet accounted for. However, if we take another $S_{k_{i-1}}$ iterates and compare $S_{k_{i+1}+2}$ with $S_{k_{i+1}+1}+S_{k_{i-1}}$, using assumption (iii), we can repeat the above arguments. This shows that $H$ is a subcontinuum.

Finally, because $\pi_{n_{i}} H \subset\left[z_{k_{i}-1}, \widehat{z}_{k_{i}-1}\right]$, Lemma 2 together with the assumption that $k_{i} \rightarrow \infty$ entail that $H$ is indeed a proper subcontinuum.

4. Arc+ray subcontinua. In this section we give sufficient conditions for a subcontinuum $H$ to be an arc+ray continuum. In [11] it is shown that $f$ being long-branched is equivalent to $Q$ being bounded. Hence, by (2), when $f$ is long-branched, the $c_{S_{k}}$ 's are bounded away from $c$.

Proposition 3. If $f$ is long-branched, then the only proper subcontinua of $\left(\left[c_{2}, c_{1}\right], f\right)$ are points and arcs.

Proof. Let $H$ be a subcontinuum with critical projections $\left\{n_{i}\right\}$. If $\left\{n_{i}\right\}$ is finite, then $H$ is an arc or point (see Lemma 1). Assume therefore that $\left\{n_{i}\right\}$ is infinite. By Lemma $3, \pi_{n_{i}} H \supset\left[c, c_{S_{k_{i+1}}}\right]$. Because $f$ is long-branched, $c_{S_{k_{i+1}}}$ is bounded away from $c$. Therefore $\left|\pi_{n} H\right| \nrightarrow 0$ and, by Lemma $2, H$ is not proper.

The next theorem makes it clear that there are non-long-branched maps with only arcs as proper subcontinua.

THEOREM 1. Let $H$ be a subcontinuum with critical projections $\left\{n_{i}\right\}$ and $\pi_{n_{i}} H=M_{n_{i}} \cup L_{n_{i}}$ as in Definition 1. If there exists $i_{0}$ such that for every $i>i_{0}$ and $i>j \geq i_{0}$ we have $c \notin \operatorname{int} f^{n_{i}-n_{j}}\left(L_{n_{i}}\right)$, then $H$ is a point, an arc, a $\sin (1 / x)$-continuum or two $\sin (1 / x)$-continua glued together at their rays.

Remark. If the subcontinuum $H$ has been constructed as in Proposition 2, then the hypotheses of Theorem 1 are met when there exists $i_{0}$ such that for every $k_{i}$ (recall: $n_{i}-n_{i-1}=S_{k_{i}}$ ) we have $S_{k_{i}+1}>n_{i}-n_{i_{0}}$.

Proof (of Theorem 1). By applying $\widehat{f}^{-\left(n_{i}-n_{i_{0}}\right)}$ we can shift $n_{i_{0}}$ into $n_{1}$. We may assume that $c$ is not periodic; in the periodic case every proper subcontinuum is an arc or a point, as one may derive from Proposition 3.

Let $\Phi:(\alpha, \beta) \rightarrow R$ be the parametrization of the dense ray constructed in Proposition 1. It suffices to consider $\operatorname{cl} \Phi\left(\left(\beta_{0}, \beta\right)\right)$, since $\operatorname{cl} \Phi\left(\left(\alpha, \alpha_{0}\right)\right)$ is similar.

Suppose that $i, j$ are such that $\beta_{i}<\beta_{i+1}=\beta_{j}<\beta_{j+1}$. Then $\Phi_{n_{i}}\left(\left[\beta_{i}, \beta_{i+1}\right]\right)=\varphi_{i}\left(\left[\beta_{i}, \beta_{i+1}\right]\right)=L_{n_{i}}$ and $\Phi_{n_{i}}\left(\left[\beta_{j}, \beta_{j+1}\right]\right)=f^{n_{j}-n_{i}}\left(L_{n_{j}}\right) \subset$ $L_{n_{i}}$. Hence, by induction, it follows that for each $m \in \mathbb{N},\left\{\Phi_{m}\left(\left[\beta_{i}, \beta_{i+1}\right]\right) \mid\right.$ $\left.n_{i} \geq m, \beta_{i} \neq \beta_{i+1}\right\}$ is a nested sequence of closed intervals. Moreover, if 
$\beta_{i}<s, t<\beta$, then

$$
\nu_{j}(\Phi(s))=\nu_{j}(\Phi(t)) \quad \text { for all } j<n_{i} .
$$

Define $\psi: \Phi\left(\left[\beta_{0}, \beta\right)\right) \rightarrow \mathbb{R}^{2}$ as

$$
\psi(x)=\left(\Phi^{-1}(x), \pi_{1} x\right) .
$$

Then $\psi\left(\Phi\left[\beta_{0}, \beta\right)\right)$ is the graph of an oscillating function $h:\left[\beta_{0}, \beta\right) \rightarrow\left[c_{2}, c_{1}\right]$, such that $h\left(\left[\beta_{i}, \beta_{i+1}\right]\right)=\Phi_{1}\left(\left[\beta_{i}, \beta_{i+1}\right]\right)$. We show that $\psi$ is uniformly continuous. For $\varepsilon>0$ let $D>0$ be such that $2^{-D}<\varepsilon / 2$. Set $D^{\prime}=\min \{i>D \mid$ $\left.\beta_{i} \neq \beta_{i+1}\right\}$ and $D^{\prime \prime}=\min \left\{i>D^{\prime} \mid \beta_{i} \neq \beta_{i+1}\right\}$. Let $\eta_{1}=\min \left\{\left|L_{n_{i}}\right| \mid i \leq D\right\}$ and $\eta_{2}=\min \left\{|A| \mid i \leq D^{\prime \prime}, A \subset M_{n_{i}}\right.$ and $\left.f^{S_{k_{i}}}(A)=L_{n_{i-1}}\right\}$. We may assume that $f$ is Lipschitz with Lipschitz constant $L \geq 2$. Let

$$
\delta=\min \left\{\frac{\varepsilon}{2}, \frac{\eta_{1}}{L^{n_{D^{\prime}} 2^{n_{D^{\prime}+1}+1}}}, \frac{\eta_{2}}{2^{n_{D^{\prime \prime}}}}\right\} .
$$

If $x, y \in \Phi\left(\left[\beta_{0}, \beta\right)\right)$ have distance less than $\delta$, then clearly $\left|\pi_{1} x-\pi_{1} y\right| \leq \delta<$ $\frac{\varepsilon}{2}$. Hence, it suffices to show that $\left|\Phi^{-1}(x)-\Phi^{-1}(y)\right| \leq \varepsilon / 2$. Assume that $\Phi^{-1}(x) \leq \Phi^{-1}(y)$, and let $i$ be such that $\Phi^{-1}(x) \in\left[\beta_{i}, \beta_{i+1}\right)$. If $i>D$, then obviously $\left|\Phi^{-1}(x)-\Phi^{-1}(y)\right|<\beta-\beta_{i} \leq 2^{-D}<\varepsilon / 2$. So assume that $i \leq D$.

We distinguish three cases:

- $\Phi^{-1}(y) \in\left[\beta_{i}, \beta_{i+1}\right)$. Since $\left.\Phi_{n_{i}}\right|_{\left[\beta_{i}, \beta_{i+1}\right]}=\left.\varphi_{i}\right|_{\left[\beta_{i}, \beta_{i+1}\right]}$ is a linear map onto $L_{n_{i}}$, it follows that

$$
\left|\Phi^{-1}(x)-\Phi^{-1}(y)\right|=\frac{\beta_{i+1}-\beta_{i}}{\left|L_{n_{i}}\right|}\left|\pi_{n_{i}} x-\pi_{n_{i}} y\right|<\frac{2^{-i}}{\eta_{1}} 2^{n_{i}} \delta<\frac{\varepsilon}{2} .
$$

- $\Phi^{-1}(y) \in\left[\beta_{j}, \beta_{j+1}\right]$, where $j$ is such that $\beta_{i+1}=\beta_{j}<\beta_{j+1}$. Now $\left.\varphi_{j}\right|_{\left[\beta_{i}, \beta_{j+1}\right]}$ is monotone, where $\left.\varphi_{j}\right|_{\left[\beta_{j}, \beta_{j+1}\right]}$ is a linear map onto $L_{n_{j}}$, and $\left.f^{n_{j}-n_{i}} \circ \varphi_{j}\right|_{\left[\beta_{i}, \beta_{i+1}\right]}=\left.\varphi_{i}\right|_{\left[\beta_{i}, \beta_{i+1}\right]}$ is a linear map onto $L_{n_{i}}$. Hence the slope of $\left.\varphi_{j}\right|_{\left[\beta_{i}, \beta_{j+1}\right]}$ is larger than

$$
\min \left\{\frac{\left|L_{n_{j}}\right|}{\beta_{j+1}-\beta_{j}}, \frac{\left|L_{n_{i}}\right|}{\left(\beta_{i+1}-\beta_{i}\right) L^{n_{j}-n_{i}}}\right\}>\frac{\eta_{1}}{L^{n_{D^{\prime}}}} .
$$

It follows that

$$
\left|\Phi^{-1}(x)-\Phi^{-1}(y)\right| \leq \frac{L^{n_{D^{\prime}}}\left|\pi_{n_{j}} x-\pi_{n_{j}} y\right|}{\eta_{1}}<\frac{L^{n_{D^{\prime}}}}{\eta_{1}} 2^{n_{j}} \delta<\frac{\varepsilon}{2} .
$$

- $\Phi^{-1}(y)>\beta_{j+1}$, where $\beta_{i+1}=\beta_{j}<\beta_{j+1}$. Let $k$ be such that $\beta_{j+1}=$ $\beta_{k}<\beta_{k+1}$. (Note that $k \leq D^{\prime \prime}$.) Because $\left\{\Phi_{n_{k}}\left(\left[\beta_{l}, \beta_{l+1}\right]\right) \mid l \geq k\right.$ and $\beta_{l} \neq$ $\left.\beta_{l+1}\right\}$ are nested intervals, $\pi_{n_{k}} y \in L_{n_{k}}$. Also, $\pi_{n_{k}} x \in M_{n_{k}}$ and $\left[\pi_{n_{k}} x, c\right]$ contains the interval $A$ that is mapped onto $L_{n_{j}}$ by $f^{n_{k}-n_{j}}$. Therefore $\left|\pi_{n_{k}} x-\pi_{n_{k}} y\right| \geq|A|>\eta_{2}$ and $d(x, y)>\eta_{2} / 2^{n_{D^{\prime \prime}}} \geq \delta$. This contradicts the choice of $x$ and $y$. 
This proves that $\psi$ is uniformly continuous and therefore we can extend $\psi$ to the closure of $R$. Because $\mathrm{cl} R$ is compact, it is homeomorphic to an arc if $\bigcap_{i \geq 1, \beta_{i} \neq \beta_{i+1}} \Phi_{1}\left(\left[\beta_{i}, \beta_{i+1}\right]\right)$ is a point, and homeomorphic to a $\sin (1 / x)$ continuum if $\bigcap_{i \geq 1, \beta_{i} \neq \beta_{i+1}} \Phi_{1}\left(\left[\beta_{i}, \beta_{i+1}\right]\right)$ is a non-degenerate interval.

Closely related to the kneading map is the Hofbauer tower [16]. Given a unimodal map $f$, the associated Hofbauer tower is the disjoint union of intervals $\left\{D_{n}\right\}_{n \geq 2}$, where $D_{2}=\left[c_{2}, c_{1}\right]$ and, for $n \geq 2$,

$$
D_{n+1}= \begin{cases}f\left(D_{n}\right) & \text { if } c \notin D_{n} \\ {\left[c_{n+1}, c_{1}\right]} & \text { if } c \in D_{n} .\end{cases}
$$

By (1) it follows that for $k \geq 2$,

$$
D_{S_{k}}=\left[c_{S_{k}}, c_{S_{Q(k)}}\right] .
$$

As the core is compact, an equivalent formulation of leo is: for every $\varepsilon>0$, there is a positive integer $N$ such that if $U$ is an open subinterval of the core with $|U| \geq \varepsilon$, then for every $n \geq N, f^{n}(U)=\left[c_{2}, c_{1}\right]$. Hence, leo, (2), and (6) give

$$
\lim _{k \rightarrow \infty} Q(k)=\infty \Rightarrow \lim _{n \rightarrow \infty}\left|D_{n}\right|=0,
$$

where $Q$ is the kneading map of $f$.

Corollary 1. Suppose $f$ is such that $Q(Q(k)+1)$ is bounded. Then every proper subcontinuum of $\left(\left[c_{2}, c_{1}\right], f\right)$ is a point, an arc, or a $\sin (1 / x)$ continuum.

Proof. The "geometric" meaning of this bound is as follows. From (6), the $S_{k}$ th level in the Hofbauer tower is $D_{S_{k}}=\left[c_{S_{k}}, c_{S_{Q(k)}}\right]$. Using (2),

$$
c_{S_{Q(k)}} \in\left(z_{Q(Q(k)+1)-1}, z_{Q(Q(k)+1)}\right] \cup\left[\widehat{z}_{Q(Q(k)+1)}, \widehat{z}_{Q(Q(k)+1)-1}\right) .
$$

Thus $Q(Q(k)+1)$ bounded means the $c_{S_{Q(k)}}$ 's are uniformly bounded away from $c$.

Suppose that $Q(Q(k)+1) \leq B$ for all $k$. Let $H$ be a subcontinuum with critical projections $\left\{n_{i}\right\}$. We may assume that $\left\{n_{i}\right\}$ is infinite; otherwise $H$ is a point or an arc by Lemma 1 . Using Lemma 2 and applying $\widehat{f}$, we may assume that

$$
\left|\pi_{n_{i}} H\right|<\left|c-z_{S_{B}}\right| \quad \text { for all } i .
$$

If for some $i, c \in f^{S_{k_{i}}}\left(\left[c, c_{S_{k_{i+1}}}\right]\right.$ ), then (use (6)) $\left[c_{S_{k_{i+1}+1}}, c_{S_{Q\left(k_{i+1}+1\right)}}\right] \subset$ $\pi_{n_{i-1}} H$, contradicting (8). Hence, Theorem 1 applies with $L_{n_{i}}=\left[c, c_{S_{k_{i+1}}}\right]$ for all $i$ and therefore $H$ is a point, an arc, or a $\sin (1 / x)$-continuum.

COROLlaRY 2. Let $H$ be a proper subcontinuum satisfying the hypotheses of Theorem 1. If additionally $\lim _{k \rightarrow \infty} Q(k)=\infty$, then $H$ is either a point or an arc. 
Proof. If $H$ is homeomorphic to a $\sin (1 / x)$-continuum or to two $\sin (1 / x)$-continua glued together, then $\bigcap_{i \geq 1, \beta_{i} \neq \beta_{i+1}} \Phi_{1}\left(\left[\beta_{i}, \beta_{i+1}\right]\right)=J$ with $J$ a non-degenerate interval. The construction of $J$ implies that $\left|D_{n}\right| \nrightarrow 0$, contradicting (7).

EXAMPLE 1. We give a map $f$ (or rather its kneading map $Q$ ) such that $f$ is not long-branched, but all proper subcontinua of $(I, f)$ are arcs or points. Let $\left\{k_{i}\right\}$ be a sequence such that $k_{i}-k_{i-1}>3$. Let $Q$ be a kneading map such that

$$
Q(k+1) \leq 10 \quad \text { if } k \notin\left\{k_{i}\right\}, \quad Q\left(k_{i}+1\right)-k_{i-1} \rightarrow \infty .
$$

As $Q$ is not bounded, $f$ is not long-branched. Let $H$ be a subcontinuum with critical projections $\left\{n_{i}\right\}$. It follows from Lemmas 2 and 3 that $n_{i}-n_{i-1} \in$ $\left\{S_{k_{j}}\right\}_{j}$ for all but finitely many $i$. For simplicity assume that $n_{i}-n_{i-1}=S_{k_{i}}$ for all $i$ (passing to a subsequence if needed). Let $i_{0}$ be such that $Q\left(k_{i}+1\right)>$ $k_{i-1}+1$ for all $i \geq i_{0}$. Then it follows by induction that $n_{i}-S_{k_{i}+1}<n_{i_{0}}$ for all $i \geq i_{0}$. In fact, $\left(n_{i}-n_{i_{0}}\right)-S_{k_{i}+1} \rightarrow-\infty$. Therefore the condition of Theorem 1 is satisfied. Write $J_{i}=\Phi_{n_{i_{0}}}\left(\left[a_{i}, a_{i+1}\right]\right)=f^{n_{i}-n_{i_{0}}}\left(\left[c, c_{S_{k_{i+1}}}\right]\right)$. Then $J_{i}$ are nested intervals, and $S_{k_{i+1}+1}-\left(n_{i}-n_{i_{0}}\right)=\min \left\{j \mid f^{j}\left(J_{i}\right) \ni c\right\}$. Because $S_{Q\left(k_{i}+1\right)}-n_{1} \rightarrow \infty,\left|J_{i-1}\right| \rightarrow 0$. As was shown in the proof of Theorem $1, H=\operatorname{cl} R$ is an arc.

THEOREM 2. Let $H$ be a subcontinuum with critical projections $\left\{n_{i}\right\}$ and $\pi_{n_{i}} H=M_{n_{i}} \cup L_{n_{i}}$ as in Definition 1. If there exists a sequence $\{i(m)\}$ such that $c \notin \operatorname{int} f^{n_{i}-n_{j}}\left(L_{n_{i}}\right)$ for every $m$, every $i>i(m)$ and every $1 \leq j<i(m)$, then $H$ is an arc+ray continuum or an arc.

Proof. We may assume that $c$ is not periodic. Let $\Phi((\alpha, \beta))$ be the dense ray in $H$ constructed in Proposition 1. Again it suffices to deal with $R \equiv \Phi\left(\left[\beta_{0}, \beta\right)\right)$. By assumption int $\Phi_{n_{j}}\left(\left[\beta_{i(m)}, \beta\right)\right) \not \supset c$ for $i(1) \leq j<i(m)$. Therefore, for all $s, t \in\left[\beta_{i(m)}, \beta\right)$ and all $n_{i(1)} \leq j<n_{i(m)}$, we have $\nu_{j}(\Phi(s))=\nu_{j}(\Phi(t))$. Let $A=\left\{x \in H \mid\right.$ there exist $t_{i} \in\left(\beta_{0}, \beta\right)$ with $\lim _{i \rightarrow \infty} t_{i}=\beta$ and $\left.\lim _{i \rightarrow \infty} \Phi\left(t_{i}\right)=x\right\}$. If $x, y \in A$, then $\nu_{j}(x)=\nu_{j}(y)$ for all $j \geq n_{i(1)}$. Therefore $\#\left\{n \mid \pi_{n}(A) \ni c\right\}<\infty$ and hence $A$ is contained in an arc component [6, Lemma 2.8]; thus $A$ is connected since $(I, f)$ is atriodic [21]. Hence, $A$ is an arc or a point by Lemma 1.

Corollary 3. Let $H$ be a subcontinuum with critical projections $\left\{n_{i}\right\}$ and $\pi_{n_{i}} H=M_{n_{i}} \cup L_{n_{i}}$ as in Definition 1. If there exists a sequence $\{i(m)\}$ such that for every $m \geq 1, i>i(m)$ and $j \geq 0$, either $n_{i}-S_{k_{i}+j} \geq n_{i(m)}$ or $n_{i}-S_{k_{i}+j}<n_{i(1)}$, then $H$ is an arc or an arc+ray continuum.

Proof. Let $R=\Phi\left(\left[\beta_{0}, \beta\right)\right)$ be as in Proposition 1 . We show that the hypothesis of Theorem 2 holds. To be precise, we show that for every $m \geq 2$, 
$i(1)<l<i(m)$ and $i \geq i(m)$,

$$
\operatorname{int} f^{n_{i}-n_{l}}\left(L_{n_{i}}\right) \not \supset c .
$$

Fix $m \geq 2$. We argue by induction on $i$. First take $i=i(m)$. By assumption $S_{Q\left(k_{i(m)+1}+1\right)}=S_{k_{i(m)+1}+1}-S_{k_{i(m)+1}}>n_{i(m)+1}-n_{i(1)}-\left(n_{i(m)+1}-n_{i(m)}\right)=$ $n_{i(m)}-n_{i(1)}$. Therefore $c_{S_{k_{i(m)+1}}} \in\left[z_{r}, \widehat{z}_{r}\right]$ for some $r$ such that $S_{r}>n_{i(m)}-$ $n_{i(1)} \geq n_{i(m)}-n_{l}$. Hence $L_{n_{i}}=\left[c, c_{S_{k_{i(m)+1}}}\right]$ and int $f^{n_{i(m)}-n_{l}}\left(L_{n_{i}}\right) \not \supset c$.

Assume now by induction that (9) holds for all $i^{\prime}, i(m) \leq i^{\prime}<i$. Assume by contradiction that $f^{n_{i}-n_{l}}\left(L_{n_{i}}\right) \ni c$. Assume also that $l$ is the largest integer $\leq i(m)$ with this property. Let $x \in f^{-\left(n_{i}-n_{l}\right)}(c) \cap L_{n_{i}}$ and let $J \ni x$ be the corresponding monotonicity interval of $f^{n_{i}-n_{l}}$. Then there exist $0<$ $a<b<n_{i}-n_{l}$ such that $c \in \partial f^{a}(J), \partial f^{b}(J)$. By the assumption on $l$, in fact $a, b \leq n_{i}-n_{i(m)}$. More precisely, $n_{i}-a=n_{i^{\prime}}$ and $b-a=S_{k_{i^{\prime}}+j}$ for some $i(m)+1<i^{\prime}<i$ and $j \geq 0$, and we get $f^{a}(J)=\left[z_{k_{i^{\prime}}+j}, c\right]$ or $\left[c, \widehat{z}_{k_{i^{\prime}}+j}\right]$. Hence

and

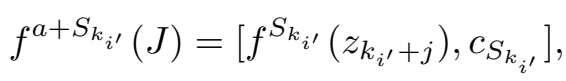

$$
c \in f^{\left(n_{i^{\prime}-1}-n_{l}\right)+\left(a+S_{k_{i^{\prime}}}\right)}(J) \subset f^{n_{i^{\prime}-1}-n_{l}} .
$$

This contradicts $\left.f^{n_{i}-n_{l}}\right|_{J}$ monotone and the induction hypothesis (9).

EXAmple 2 . We call $H$ an $M W$-continuum if it is homeomorphic to the closure of the graph $\{(x, h(1 / x)) \mid x \in(0,1]\}$ where $h(x)=\sin x+\sin 3 x$. The shape of the graph explains the name; cf. the M-continuum in [21, Exercise 3.32]. We give the kneading map of a map $f$ such that $(I, f)$ has an MW-continuum as proper subcontinuum. Suppose $\left\{k_{i}\right\}_{i}$ is an integer sequence, and $Q$ satisfies:

$$
\begin{array}{rlrl}
Q\left(k_{3}+1\right) & =k_{2}, & \\
Q\left(k_{4}+1\right) & =k_{3}, \quad Q\left(k_{4}+2\right)=k_{2}, & \\
Q\left(k_{i}+1\right) & =k_{i-1}, \quad Q\left(k_{i}+2\right)=k_{i-2}+2, & \\
Q\left(k_{i}+3\right)=5, & \text { and } Q\left(k_{i}+4\right) \geq i & & \text { if } i>4 \text { is even, } \\
Q\left(k_{i}+1\right) & =k_{i-1}+2, \quad Q\left(k_{i}+2\right) \geq i & \text { if } i>4 \text { is odd. }
\end{array}
$$

Let $\left\{n_{i}\right\}_{i}$ be such that $n_{i}-n_{i-1}=S_{k_{i}}$, and let $H$ be the subcontinuum with critical projections $\left\{n_{i}\right\}_{i}$ as constructed in Proposition 2.

Let $\Phi$ be the parametrization of the ray $R$. We sketch why $H=\operatorname{cl} R$ is an MW-continuum. First observe that

$$
n_{i}-n_{1}= \begin{cases}S_{k_{i}+2} & \text { if } i>4 \text { is even, } \\ S_{k_{i}+1} & \text { if } i>4 \text { is odd. }\end{cases}
$$

This can be shown by induction. Theorem 2 applies (with $i(m)=2 m$ ) since $S_{k_{i}+1}=n_{i}-n_{i-2}$ for $i>4$ even. Note also that (cf. Proposition 2) 
$L_{n_{i-1}}=\left[c, c_{S_{k_{i}}}\right]$ for each $i$. Therefore $L_{n_{i-1}}=\varphi_{i-1}\left(\left[\beta_{i-1}, \beta_{i}\right]\right)$ for all $i$. In particular, $\beta_{i-1}<\beta_{i}$ while $\alpha_{i-1}=\alpha_{i}$ for each $i \geq 1$. We finish the sketch by computing $\left.\pi_{n_{1}} \circ \Phi\right|_{\left[\beta_{i-1}, \beta_{i}\right]}$ for each $i$ (see Figure 1). We use (2) repeatedly here.

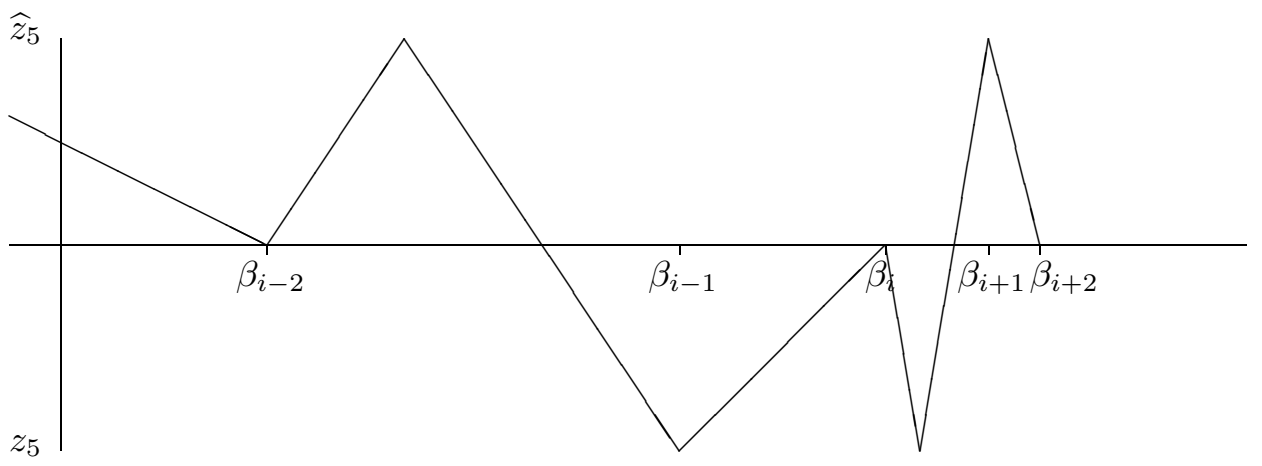

Fig. 1. $\pi_{n_{1}} \circ \Phi:(\alpha, \beta) \rightarrow I, i$ is odd

- $i$ is odd. Now $n_{i-1}-n_{1}=S_{k_{i}+1}-S_{k_{i}}=S_{k_{i-1}+2}$ and $f^{n_{i-1}-n_{1}}$ maps $L_{n_{i-1}}$ monotonically onto $\left[c_{S_{k_{i-1}+2}}, c_{S_{k_{i}+1}}\right]$. Because $Q\left(k_{i}+2\right) \geq i \rightarrow \infty$, we have $c_{S_{k_{i}+1}} \rightarrow c$ as $i \rightarrow \infty$. However, $Q\left(k_{i-1}+3\right)=5$, and because $Q\left(k_{i-1}+4\right) \geq i-1 \rightarrow \infty$, we can derive $c_{S_{k_{i-1}+2}} \rightarrow z_{5}$ or $\widehat{z}_{5}$ as $i \rightarrow \infty$.

- $i$ is even. This time $f^{S_{k_{i-1}}}$ maps $L_{n_{i-1}}$ onto $\left[c_{S_{k_{i-1}}}, c_{S_{k_{i}}+S_{k_{i-1}}}\right]=$ $\left[c_{S_{k_{i-1}}}, c_{S_{k_{i}+1}}\right] \ni c$. Afterwards, $f^{n_{i-2}-n_{1}} \operatorname{maps}\left[c, c_{S_{k_{i-1}}}\right]=L_{n_{i-2}}$ onto $\left[c_{S_{k_{i-2}+2}}, c_{S_{k_{i-1}+1}}\right] \supset\left[z_{5}, c\right]$ or $\left[c, \widehat{z}_{5}\right]$ as in the previous case. The other component $\left[c, c_{S_{k_{i}+1}}\right]$ is mapped monotonically onto $\left[c_{S_{k_{i-2}+2}}, c_{S_{k_{i}+1}+S_{k_{i-2}+2}}\right]=$ $\left[c_{S_{k_{i-2}+2}}, c_{S_{k_{i}+2}}\right]$. Because $Q\left(k_{i-2}+3\right)=Q\left(k_{i}+3\right)=5$, this interval contains $\left[z_{5}, \widehat{z}_{5}\right]$. (Because both $Q\left(k_{i-2}+4\right)$ and $Q\left(k_{i}+3\right) \rightarrow \infty$ the intervals $\left[c_{S_{k_{i-2}+2}}, c_{S_{k_{i}+2}}\right]$ converge to $\left[z_{5}, \widehat{z}_{5}\right]$ as $i \rightarrow \infty$.)

This proves Figure 1.

As remarked in the preliminaries, if maps $f$ and $g$ are topologically conjugate, then their inverse limit spaces $(I, f)$ and $(I, g)$ are homeomorphic. Hence, in the next example one may assume that $f$ is a tent map.

EXAMPLE 3. Let $f$ have the Fibonacci combinatorics, i.e., $Q(k)=$ $\max \{0, k-2\}$ (see e.g. [19]). The cutting times $\left\{S_{k}\right\}_{k \geq 0}$ are precisely the Fibonacci numbers: $S_{0}=1, S_{1}=2$, and $S_{k+1}=S_{k}+S_{k-1}$. An image $c_{n}$ is a closest return of $c$ provided $c_{j} \notin\left[c_{n}, \widehat{c}_{n}\right]$ for $0<j<n$. For the map $f$, closest returns occur iff $n \in\left\{S_{k}\right\}_{k \geq 0}$. We construct two proper subcontinua $E$ and $F$ of $(I, f)$ with the following properties: 
(i) The continuum $F$ is an arc and $F \subset E$.

(ii) Let $R=\Phi([0, \infty))$ be the ray from Proposition 1 for the subcontinuum $E$. Then $R \cap F=\emptyset$ and $\lim _{t \rightarrow \infty} d(\Phi(t), F)=0$.

(iii) The continuum $E$ is an arc+ray continuum.

(iv) Let $B$ be a non-arc proper subcontinuum of $(I, f)$. Then there exists an integer $t$ and an arc $A$ such that $\widehat{f}^{t}(B) \subset E \cup A$.

First we construct $E$. Set $k_{i}=i-1$ and $n_{i}-n_{i-1}=S_{k_{i}}$ for $i \geq 2$. Let $\pi_{n_{i}} E=\left[b_{i}, c_{S_{k_{i+1}}}\right]$ for $i \geq 1, b_{1}=c_{1}$, and $M_{n_{i}}=\left[c, b_{i}\right]$ for $i \geq 1$. It follows from (2) and (6) that $E$ can be constructed as stated; see Figure 2.

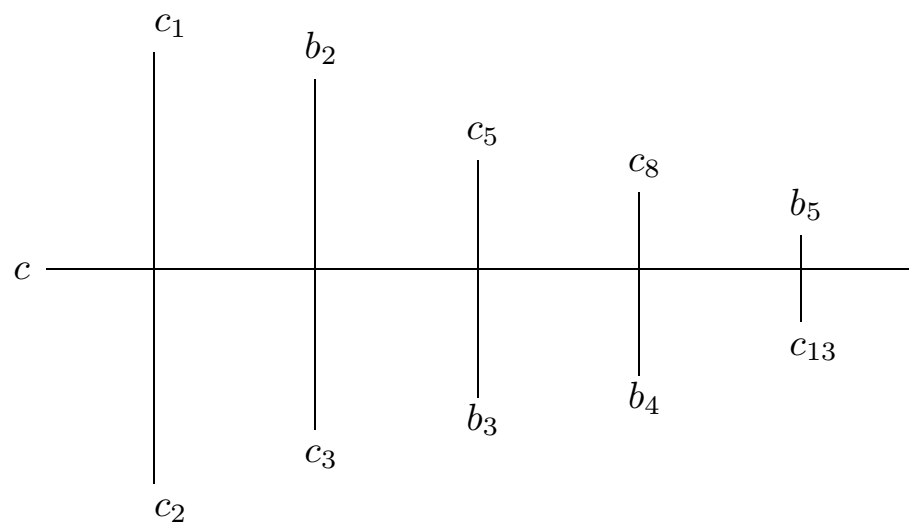

Fig. 2. Critical projections $\pi_{n_{i}} E$ for $1 \leq i \leq 5$

Next we construct $F$. Again, $k_{i}=i-1$ and $n_{i}-n_{i-1}=S_{k_{i}}$ for $i \geq 2$. Set $\pi_{n_{i}} F=\left[c_{S_{i+2}}, c_{S_{k_{i+1}}}\right]$, for $i \geq 1$; here $M_{n_{i}}=\left[c, c_{S_{k_{i+1}}}\right]$ for all $i$. By construction, $F \subset E$; see Figure 3. For all $i>j$ we have int $f^{n_{i}-n_{j}}\left(L_{n_{i}}\right) \not \supset c$ and therefore, by Theorem $1, F$ is an arc.

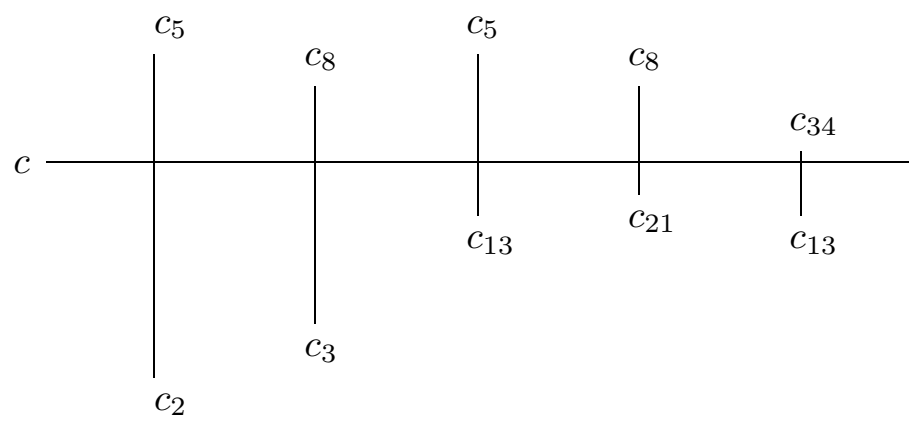

Fig. 3. Critical projections $\pi_{n_{i}} F$ for $1 \leq i \leq 5$ 
It follows from the proof of Proposition 1 that the points $x=\left(x_{1}, x_{2}, \ldots\right)$ $\in R$ are precisely those $x \in(I, f)$ for which there exists $l \in \mathbb{N}$ with $x_{n_{i}} \in M_{n_{i}}^{E}$ for all $n_{i} \geq l$. For $E, M_{n_{i}}^{E}=\left[c, b_{i}\right]$ all $i$. For $F, M_{n_{i}}^{F}=\left[c, c_{S_{k_{i+1}}}\right]$ all $i$. Thus $M_{n_{i}}^{E} \cap M_{n_{i}}^{F}=\{c\}$. Hence the second property holds.

For the third property, let $x=\left(x_{1}, x_{2}, \ldots\right) \in E \backslash F$; then there exists an $i$ such that $x_{n_{i}} \notin F_{n_{i}}$. Hence, $x_{n_{j}} \notin F_{n_{j}}$ for all $j \geq i$. But for each $j$, $F_{n_{j}} \supset L_{n_{j}}^{E}$. Thus, $x_{n_{j}} \in M_{n_{j}}^{E}$ for all $j \geq i$. Therefore $x \in R$.

For the fourth property, assume that $B$ is a proper subcontinuum of $(I, f)$ and that $B$ is not an arc. Let $\left\{m_{i}\right\}$ be its critical projections with $m_{i}-m_{i-1}=S_{l_{i}}$.

Claim 1. We have $l_{i+1}>l_{i}$ for $i \geq 1$.

Proof. Because $\pi_{m_{i}} B \supset\left[c, c_{S_{l_{i+1}}}\right]$ and $m_{i-1}$ is the previous critical return, $f^{S_{l_{i}}}=f^{m_{i}-m_{i-1}}$ is monotone on $\left[c, c_{S_{l_{i+1}}}\right]$. But by $(2)$ and the fact that $Q(k)=k-2$, we have $c_{S_{l_{i+1}}} \notin\left[z_{l_{i+1}-1}, \widehat{z}_{l_{i+1}-1}\right]$. Therefore, $S_{l_{i}} \leq$ $S_{l_{i+1}-1}$, as was claimed. In particular, as $k_{i}=i-1, l_{i} \geq k_{i}$ for all $i \geq 2$.

Claim 2. For $i \geq 1,\left[c_{S_{l_{i+1}}}, c\right] \subset \pi_{n_{i}} E$.

Pr o of. The closest returns $c_{n}$ to $c$ occur when $n$ is a cutting time $[11,19]$. Since $l_{i+1} \geq k_{i+1}$, we get $\left|c_{S_{l_{i+1}}}-c\right| \leq\left|c_{S_{k_{i+1}}}-c\right|$. Now $\pi_{n_{i}} E=\left[c_{S_{k_{i+1}}}, b_{i}\right]$ and because $f^{S_{k_{i}}}\left(\left[c, c_{S_{k_{i+1}}}\right]\right)$ is properly contained in $\pi_{n_{i-1}} E=f^{S_{k_{i}}}\left(\left[c, b_{i}\right]\right)$, we get $\left|b_{i}-c\right|>\left|c_{S_{k_{i+1}}}-c\right|$. This proves Claim 2 .

Claim 3. Suppose that $l_{i}=k_{i} \equiv i-1$, or that $l_{i} \geq i+1$, for some $i$. Then $\pi_{m_{i-1}} B \subset \pi_{n_{i-1}} E$.

Proof. Suppose first that $l_{i}=k_{i}=i-1$ for some $i$. Because $k_{j} \equiv j-1$, $l_{j+1}>l_{j}$ for all $j$, and $l_{2} \geq 1$, we obtain $l_{j}=k_{j}$ for all $2 \leq j \leq i$. As $\left[c, b_{2}\right]$ is a maximal interval of monotonicity of $f^{n_{2}}$, and by Claim 2 , it follows that $\pi_{m_{i-1}} B \subset \pi_{n_{i-1}} E$.

Secondly, if $l_{i} \geq i+1$, then because $m_{i}$ and $m_{i-1}$ are consecutive critical projections for $B$ and $m_{i}-m_{i-1}=S_{l_{i}}$, it follows that $\pi_{m_{i-1}} B \subset$ $D_{S_{l_{i}}}=\left[c_{S_{j}}, c_{S_{Q(j)}}\right]=\left[c_{S_{j}}, c_{S_{j-2}}\right]$ for some $j \geq i+1$. Recall that $\pi_{n_{i-1}} E=$ $\left[c_{S_{k_{i}}}, b_{i-1}\right]=\left[c_{S_{i-1}}, b_{i-1}\right]$ and that $\left|b_{i-1}-c\right|>\left|c_{S_{i-1}}-c\right|$. Because closest returns occur only at cutting times, we get $\left|c_{S_{j}}-c\right|<\left|c_{S_{j-2}}-c\right| \leq\left|c_{S_{i-1}}-c\right|<$ $\left|b_{i-1}-c\right|$, and thus $\pi_{m_{i-1}} B \subset \pi_{n_{i-1}} E$. Claim 3 is now complete.

Recall that $l_{i} \geq k_{i}=i-1$ for all $i$ and hence $l_{i} \geq i-1$ for all $i$. If for infinitely many $i$ we have $l_{i} \in\{i-1, i+1, i+2, \ldots\}$, then, by Claim 3, $\pi_{m_{i}} B \subset \pi_{n_{i}} E$ for infinitely many $i$ and hence $B \subset E$.

Lastly, assume that $l_{i} \in\{i-1, i+1, i+2, \ldots\}$ for only finitely many $i$. Set $i_{0}=\min \left\{j \mid i \geq j \Rightarrow l_{i}=i\right\}, n=n_{i_{0}+1}$, and $m=m_{i_{0}}$. Let $A_{m_{i_{0}}}=\pi_{m_{i_{0}}} B \backslash \pi_{n_{i_{0}}+1} E$. If $A_{m_{i_{0}}}=\emptyset$, then $\pi_{m_{i}} B \subset \pi_{n_{i+1}} E$ for all $i \geq i_{0}$ 
and therefore for all $i \in \mathbb{N}$. In this case, $B \subset \widehat{f}^{n-m}(E)$. If $A_{m_{i_{0}}} \neq \emptyset$, then for every $i \geq i_{0}, A_{m_{i}}=\pi_{m_{i}} B \backslash \pi_{n_{i+1}} E$ is such that $f^{m_{i}-m_{i_{0}}}\left(A_{m_{i}}\right)=$ $A_{m_{i_{0}}}$. Because $\left\{m_{i}\right\}$ are the only critical projections, in fact $f^{m_{i}-m_{i_{0}}} \mid A_{m_{i}}$ is monotone. Let $A \subset(I, f)$ be such that $\pi_{m_{i}} A=A_{m_{i}}$ for $i \geq i_{0}$. By Lemma $1, A$ is an arc and $B \subset \widehat{f}^{n-m}(E) \cup A$.

5. Subcontinua. In this section we study subcontinua that are more complicated than arc+ray continua. In [3] a method was demonstrated to construct subcontinua that are homeomorphic to the entire inverse limit space of some unimodal map. A key observation is that the map $f^{S_{k_{i}}}$ : $\pi_{n_{i}} H \rightarrow \pi_{n_{i-1}} H$ is a rescaling of a unimodal map. Under the assumption that the rescalings of $f^{S_{k_{i}}}: \pi_{n_{i}} H \rightarrow \pi_{n_{i-1}} H$ are sufficiently close to a fixed unimodal map $g: J \rightarrow J$ (where the sufficient closeness depends on $i), H$ was proven to be homeomorphic to $(J, g)$. In Proposition 4 , we give two conditions, one combinatorial and the other analytic, on sequences of unimodal maps $\left\{f_{i}\right\}$ and $\left\{g_{i}\right\}$ to guarantee that $\left(I_{i}, f_{i}\right)$ and $\left(J_{i}, g_{i}\right)$ are homeomorphic. We then relate the combinatorial condition of Proposition 4 to the properties of cutting times. Lastly, in Theorem 3, given a finite or infinite sequence $\mathcal{F}$ of unimodal maps with periodic turning points, we provide a unimodal map $g$ such that the non-arc+ray subcontinua of $(I, g)$ are precisely the subcontinua from the collection $\{(I, f)\}_{f \in \mathcal{F} \text {. }}$

Based on the construction in Theorem 3, we conclude that there are uncountably many non-homeomorphic unimodal inverse limit spaces. This uncountability is also established by the authors of [5]; however, they use renormalizable maps whereas our maps need not be renormalizable.

Throughout this section for any $\left(I_{i}, f_{i}\right)$ we assume each $f_{i}: I_{i} \rightarrow I_{i-1}$ is onto and unimodal. Similar to Definition 1, let $M_{i}$ be the closure of the component of $I_{i} \backslash\{c\}$ such that $f_{i}\left(M_{i}\right)=I_{i-1}$, and let $L_{i}$ be the closure of the remaining component. Again, if there is a choice, set $L_{i}$ to be the component containing $f_{i+1}(c)$. For convenience, let $c$ represent the turning point of each $f_{i}$, allowing $c$ to vary with $i$.

Let $\left(I_{i}, f_{i}\right)$ be given and for $i \geq 1$ and $z \in I_{i}$ set

$$
\widehat{\nu}_{i}(z)= \begin{cases}1 & \text { if } z \in\left(c, f_{i+1}(c)\right], \\ * & \text { if } z=c, \\ 0 & \text { if } z \notin\left[c, f_{i+1}(c)\right] .\end{cases}
$$

For $x=\left(x_{1}, x_{2}, \ldots\right) \in\left(I_{i}, f_{i}\right)$ set

$$
\widehat{\nu}(x)=\left(\widehat{\nu}_{1}\left(x_{1}\right), \widehat{\nu}_{2}\left(x_{2}\right), \ldots\right),
$$

and define

$$
T(x)=\left\{y \in\left(I, f_{i}\right) \mid \widehat{\nu}(x)=\widehat{\nu}(y)\right\}
$$


Set

$$
T_{1}(x)=\left\{\pi_{1}(y) \mid y \in T(x)\right\} .
$$

As in the proof of Lemma 1, each $T(x)$ is either a point or an arc and hence each $T_{1}(x)$ is a point or a non-degenerate subinterval of $I_{1}$. For convenience let $\left.\widehat{\nu}\right|_{i}(x)=\widehat{\nu}_{1}\left(x_{1}\right), \ldots, \widehat{\nu}_{i}\left(x_{i}\right)$ for $x \in\left(I_{i}, f_{i}\right)$ and $\left.\widehat{\nu}\right|_{i}(z)=$ $\widehat{\nu}_{1}\left(f_{i, 1}(z)\right), \ldots, \widehat{\nu}_{i-1}\left(f_{i}(z)\right), \widehat{\nu}_{i}(z)$ for $z \in I_{i}$. For $i \geq 1$ set

$$
C_{i}=\partial I_{i} \cup \bigcup_{1 \leq j \leq i} f_{i, j}^{-1}(c) \subset I_{i} .
$$

Each $C_{i}$ defines a partition of $I_{i}$, which we denote by $\mathcal{P}_{i}$. When more than one inverse limit space is being discussed, we use $C_{i}^{f}$ and $C_{i}^{g}$ etc.

Definition 2. We say $\left(I_{i}, f_{i}\right)$ is combinatorially equivalent to $\left(J_{i}, g_{i}\right)$, written $\left(I_{i}, f_{i}\right) \approx\left(J_{i}, g_{i}\right)$, provided that for each $i$ there exists a monotone bijection $h_{i}: C_{i}^{f} \rightarrow C_{i}^{g}$ such that $\left.\widehat{\nu}\right|_{i}(\alpha)=\left.\widehat{\nu}\right|_{i}\left(h_{i}(\alpha)\right)$ for each $\alpha \in C_{i}^{f}$ and such that $h_{i}\left(c^{f}\right)=c^{g}$.

In [6, Corollary 1.1] a different definition of combinatorial equivalence is given.

For ease of notation, in the statement and proof of Proposition 4 we assume that $f_{i}(c) \in M_{i-1}^{f}$ and $g_{i}(c) \in M_{i-1}^{g}$ for all $i$, i.e., that $I_{i}=\left[f_{i+2, i}(c)\right.$, $\left.f_{i+1, i}(c)\right]$ and $J_{i}=\left[g_{i+2, i}(c), g_{i+1, i}(c)\right]$ for all $i$. Assuming no $f_{i}$ or $g_{i}$ is the full unimodal map, we see that $f_{i+3, i}(c)$ and $g_{i+3, i}(c)$ are in the interior of $I_{i}$ respectively $J_{i}$.

Proposition 4. Let $\left(I_{i}, f_{i}\right)$ and $\left(J_{i}, g_{i}\right)$ be given such that

$$
\left(I_{i}, f_{i}\right) \approx\left(J_{i}, g_{i}\right) .
$$

Suppose that $a_{i-3}^{f}, b_{i-3}^{f} \in C_{i-3}^{f}$ are such that $f_{i, i-3}(c) \in\left[a_{i-3}^{f}, b_{i-3}^{f}\right]=\operatorname{cl} P_{i-3}^{f}$ for some $P_{i-3}^{f} \in \mathcal{P}_{i-3}^{f}$. Write $A_{i-3}^{f}=f_{i-3,1}\left(a_{i-3}^{f}\right)$ and $B_{i-3}^{f}=f_{i-3,1}\left(b_{i-3}^{f}\right)$. Let $a_{i-3}^{g}, b_{i-3}^{g}, A_{i-3}^{g}$ and $B_{i-3}^{g}$ be the corresponding points for $\left(J_{i}, g_{i}\right)$. If for all $i$ sufficiently large

$$
\begin{aligned}
\left(1-\frac{1}{i^{2}}\right) \frac{\left|B_{i-3}^{f}-f_{i, 1}(c)\right|}{\left|B_{i-3}^{f}-A_{i-3}^{f}\right|} & \leq \frac{\left|B_{i-3}^{g}-g_{i, 1}(c)\right|}{\left|B_{i-3}^{g}-A_{i-3}^{g}\right|} \\
& \leq\left(1+\frac{1}{i^{2}}\right) \frac{\left|B_{i-3}^{f}-f_{i, 1}(c)\right|}{\left|B_{i-3}^{f}-A_{i-3}^{f}\right|}
\end{aligned}
$$

then $\left(I_{i}, f_{i}\right)$ is homeomorphic to $\left(J_{i}, g_{i}\right)$.

Proof. We first show that for each $x \in\left(I_{i}, f_{i}\right)$ there exists $y \in\left(J_{i}, g_{i}\right)$ such that $\widehat{\nu}(x)=\widehat{\nu}(y)$; similarly it will follow that for each $y \in\left(J_{i}, g_{i}\right)$ there exists $x \in\left(I_{i}, f_{i}\right)$ such that $\widehat{\nu}(y)=\widehat{\nu}(x)$. 
Let $x=\left(x_{1}, x_{2}, \ldots\right) \in\left(I_{i}, f_{i}\right)$. If $\widehat{\nu}(x)$ contains at least one $*$, then, from $(10)$, there is a unique $y \in\left(J_{i}, g_{i}\right)$ such that $\widehat{\nu}(y)=\widehat{\nu}(x)$. Hence, we assume that $\widehat{\nu}(x)$ does not contain an $*$. Then for each $i \geq 2$ we can uniquely choose, using (10), a $P_{i}^{g} \in \mathcal{P}_{i}^{g}$ such that $\left.\widehat{\nu}\right|_{i}(x)=\left.\widehat{\nu}\right|_{i}(z)$ for each $z \in P_{i}^{g}$. Set $J=\bigcap_{i \geq 2} g_{i, 1}\left(P_{i}^{g}\right)$; note that the sequence $\left\{g_{i, 1}\left(P_{i}^{g}\right)\right\}_{i \geq 2}$ is nested. There exists a $y \in\left(J_{i}, g_{i}\right)$ such that $\widehat{\nu}(y)=\widehat{\nu}(x) \Leftrightarrow J \neq\{c\}$. But, by $(11), J=\{c\} \Leftrightarrow \bigcap_{i>2} f_{i, 1}\left(h_{i}^{-1} P_{i}^{g}\right)=\{c\} \Rightarrow x_{1}=c$. Hence, $J \neq\{c\}$ as $x_{1} \neq c$ since there is no $*$ in $\widehat{\nu}(x)$.

We have shown that for each $x \in\left(I_{i}, f_{i}\right)$, there exists $y \in\left(J_{i}, g_{i}\right)$ such that $\widehat{\nu}(x)=\widehat{\nu}(y)$. If $x_{i} \in C_{i}^{f}$ for some $i$, then the point $y$ is unique. Otherwise, we need to show that $T_{1}^{f}(x)$ and $T_{1}^{g}(y)$ are comparable in size. So assume from now on that $x_{i} \neq c$ for all $i \geq 1$, and that $\widehat{\nu}(x)=\widehat{\nu}(y)$.

Let $i_{0}$ be such that (11) holds for all $i \geq i_{0}$. Let

$$
\begin{aligned}
& K^{-}=\min \left\{\frac{\left|g_{i_{0}, 1}\left(h_{i_{0}}(P)\right)\right|}{\left|f_{i_{0}, 1}(P)\right|} \mid P \in \mathcal{P}_{i_{0}}^{f}\right\}, \\
& K^{+}=\max \left\{\frac{\left|g_{i_{0}, 1}\left(h_{i_{0}}(P)\right)\right|}{\left|f_{i_{0}, 1}(P)\right|} \mid P \in \mathcal{P}_{i_{0}}^{f}\right\},
\end{aligned}
$$

where $h_{i_{0}}$ is from Definition 2. For each $i$ let $P_{i}^{f}(x) \in \mathcal{P}_{i}^{f}$ be such that $x_{i} \in P_{i}^{f}(x)$. Let $P_{i}^{g}(y)$ be the same object for $y$. In fact, $h_{i}\left(\partial P_{i}^{f}\right)=\partial P_{i}^{g}(y)$.

Let $i_{1}=i_{1}(x)$ be the largest integer greater than $i_{0}$ such that

$$
f_{i_{1}-2, i_{0}}\left(P_{i_{1}-2}^{f}(x)\right) \neq P_{i_{0}}^{f}(x) .
$$

This means that $f_{i_{1}+1, i_{1}-2}(c) \in \operatorname{int} P_{i_{1}-2}^{f}(x)$ and $c \in \partial P_{i_{1}+1}^{f}(x)$. In particular, $f_{i_{1}, i_{1}-3}\left(P_{i_{1}}^{f}(x)\right)=\left(f_{i_{1}, i_{1}-3}(c), b_{i_{1}-3}^{f}\right)$ and $g_{i_{1}, i_{1}-3}\left(P_{i_{1}}^{g}(y)\right)=\left(g_{i_{1}, i_{1}-3}(c)\right.$, $\left.b_{i_{1}-3}^{g}\right)$. Thus, from

$$
\begin{aligned}
\frac{\left|g_{i_{1}, 1}\left(P_{i_{1}}^{g}(y)\right)\right|}{\left|f_{i_{1}, 1}\left(P_{i_{1}}^{f}(x)\right)\right|}= & \frac{\left|g_{i_{1}, 1}\left(P_{i_{1}}^{g}(y)\right)\right|}{\left|g_{i_{1}-3,1}\left(P_{i_{1}-3}^{g}(y)\right)\right|} \\
& \cdot \frac{\left|g_{i_{1}-3,1}\left(P_{i_{1}-3}^{g}(y)\right)\right|}{\left|f_{i_{1}-3,1}\left(P_{i_{1}-3}^{f}(x)\right)\right|} \cdot \frac{\left|f_{i_{1}-3,1}\left(P_{i_{1}-3}^{f}(x)\right)\right|}{\left|f_{i_{1}, 1}\left(P_{i_{1}}^{f}(x)\right)\right|},
\end{aligned}
$$

it follows by (11) that

$$
\left(1-\frac{1}{i_{1}^{2}}\right) \frac{\left|g_{i_{0}, 1}\left(P_{i_{0}}^{g}(y)\right)\right|}{\left|f_{i_{0}, 1}\left(P_{i_{0}}^{f}(x)\right)\right|} \leq \frac{\left|g_{i_{1}, 1}\left(P_{i_{1}}^{g}(y)\right)\right|}{\left|f_{i_{1}, 1}\left(P_{i_{1}}^{f}(x)\right)\right|} \leq\left(1+\frac{1}{i_{1}^{2}}\right) \frac{\left|g_{i_{0}, 1}\left(P_{i_{0}}^{g}(y)\right)\right|}{\left|f_{i_{0}, 1}\left(P_{i_{0}}^{f}(x)\right)\right|} .
$$

Next find $i_{2}=i_{2}(x)>i_{1}$ maximal such that $f_{i_{2}-2, i_{1}}\left(P_{i_{2}}^{f}(x)\right) \neq P_{i_{1}}^{f}(x)$ and repeat the argument. In this way we find for every $i_{n}(x)>i_{0}$, and in fact for every $i \geq i_{0}$, 


$$
\begin{aligned}
\prod_{m \geq i_{0}}\left(1-\frac{1}{m^{2}}\right) \frac{\left|g_{i_{0}, 1}\left(P_{i_{0}}^{g}(y)\right)\right|}{\left|f_{i_{0}, 1}\left(P_{i_{0}}^{f}(x)\right)\right|} & \leq \frac{\left|g_{i, 1}\left(P_{i}^{g}(y)\right)\right|}{\left|f_{i, 1}\left(P_{i}^{f}(x)\right)\right|} \\
& \leq \prod_{m \geq i_{0}}\left(1+\frac{1}{m^{2}}\right) \frac{\left|g_{i_{0}, 1}\left(P_{i_{0}}^{g}(y)\right)\right|}{\left|f_{i_{0}, 1}\left(P_{i_{0}}^{f}(x)\right)\right|}
\end{aligned}
$$

Note that this is also true if $i_{n}(x)$ does not exist for some $n$, because in that case $f_{i, i_{n-1}}\left(P_{i}^{f}(x)\right)=P_{i_{n-1}}^{f}(x)$ for all $i \geq i_{n-1}$. Set

$$
K_{1}=K^{-} \prod_{m \geq i_{0}}\left(1-\frac{1}{m^{2}}\right) \quad \text { and } \quad K_{2}=K^{+} \prod_{m \geq i_{0}}\left(1+\frac{1}{m^{2}}\right) .
$$

Because (12) is uniform in $i$, this gives in the limit, i.e. for $T_{1}^{f}(x)=$ $\bigcap_{i} f_{i, 1}\left(P_{i}^{f}(x)\right)$ and $T_{1}^{g}(y)=\bigcap_{i} g_{i, 1}\left(P_{i}^{g}(y)\right)$,

$$
K_{1}\left|T_{1}^{f}(x)\right| \leq\left|T_{1}^{g}(y)\right| \leq K_{2}\left|T_{1}^{f}(x)\right| .
$$

We now have the following two possibilities. Let $x=\left(x_{1}, x_{2}, \ldots\right) \in$ $\left(I_{i}, f_{i}\right)$. If $\widehat{\nu}(x)$ contains at least one $*$, then there exists a unique $y=$ $\left(y_{1}, y_{2}, \ldots\right) \in\left(J_{i}, g_{i}\right)$ such that $\widehat{\nu}(x)=\widehat{\nu}(y)$. In this case, the homeomorphism will simply take $x$ to $y$, i.e. $h(x)=y$. If $\widehat{\nu}(x)$ contains no $*$, then $T_{1}(y)$ is independent of the $y$ chosen with $\widehat{\nu}(x)=\widehat{\nu}(y)$. In this case we define the homeomorphism as follows. Let $\psi_{x}: \operatorname{cl} T_{1}^{f}(x) \rightarrow \operatorname{cl} T_{1}^{g}(y)$ be linear onto and orientation preserving (i.e. the endpoint of $T_{1}^{f}(x)$ closest to $f_{2}(c)$ is mapped onto the endpoint of $T_{1}^{g}(y)$ closest to $g_{2}(c)$ ). Note that the slope of $\psi_{x}$ lies between $K_{1}$ and $K_{2}$. Now define $h(x)$ as $h(x)=z$, where $z \in\left(J_{i}, g_{i}\right)$ is such that $\widehat{\nu}(x)=\widehat{\nu}(z)$ and $\pi_{1}(z)=\psi_{x}\left(\pi_{1}(x)\right)$.

Finally, we show that $h$ and $h^{-1}$ are continuous. Suppose $\left\{x^{j}\right\}_{j} \subset\left(I_{i}, f_{i}\right)$ and $x^{j} \rightarrow x$. Then $\widehat{\nu}_{i}\left(x_{i}^{j}\right) \rightarrow \widehat{\nu}_{i}\left(x_{i}\right)$ whenever $\widehat{\nu}_{i}\left(x_{i}\right) \neq *$. Let $y^{j}=h\left(x^{j}\right)$ and $y=h(x)$. Because $\widehat{\nu}\left(x^{j}\right)=\widehat{\nu}\left(y^{j}\right)$ and $\widehat{\nu}(x)=\widehat{\nu}(y)$, it suffices to prove that $y_{1}^{j} \rightarrow y_{1}$.

Pick $n \geq i_{0}$ large and suppose that $x_{n} \in \operatorname{cl} P_{n}^{f}(x)=\left[a_{n}^{f}, b_{n}^{f}\right]$, where $P_{n}^{f}(x) \in \mathcal{P}_{n}^{f}$. Write $\operatorname{cl} T_{1}^{f}(x)=\left[p^{f}(x), q^{f}(x)\right]$ and $\operatorname{cl} T_{1}^{g}(y)=\left[p^{g}(y), q^{g}(y)\right]$. We have

$$
\left|y_{1}-A_{n}^{g}\right|=\frac{\left|y_{1}-p^{g}(y)\right|}{\left|q^{g}(y)-p^{g}(y)\right|}\left|q^{g}(y)-p^{g}(y)\right|+\left|p^{g}(y)-A_{n}^{g}\right| .
$$

By definition of $\psi_{x}$,

$$
\frac{\left|y_{1}-p^{g}(y)\right|}{\left|q^{g}(y)-p^{g}(y)\right|}=\frac{\left|x_{1}-p^{f}(x)\right|}{\left|q^{f}(x)-p^{f}(x)\right|}
$$


The arguments leading to (12) also prove that for all $i \geq n$,

$$
\prod_{m \geq n}\left(1-\frac{1}{m^{2}}\right) \frac{\left|f_{i, 1}\left(P_{i}^{f}(x)\right)\right|}{\left|B_{n}^{f}-A_{n}^{f}\right|} \leq \frac{\mid g_{i, 1}\left(P_{i}^{g}(y) \mid\right.}{\left|B_{n}^{g}-A_{n}^{g}\right|} \leq \prod_{m \geq n}\left(1+\frac{1}{m^{2}}\right) \frac{\left|f_{i, 1}\left(P_{i}^{f}(x)\right)\right|}{\left|B_{n}^{f}-A_{n}^{f}\right|}
$$

whence for the limit $\left[p^{g}(y), q^{g}(y)\right]=\operatorname{cl}\left(\bigcap_{i} g_{i, 1} P_{i}^{g}(y)\right)$,

$$
\begin{aligned}
\prod_{m \geq n}\left(1-\frac{1}{m^{2}}\right) \frac{\left|q^{f}(x)-q^{f}(x)\right|}{\left|B_{n}^{f}-A_{n}^{f}\right|} & \leq \frac{\left|q^{g}(y)-p^{g}(y)\right|}{\left|B_{n}^{g}-A_{n}^{g}\right|} \\
& \leq \prod_{m \geq n}\left(1+\frac{1}{m^{2}}\right) \frac{\left|q^{f}(x)-p^{f}(x)\right|}{\left|B_{n}^{f}-A_{n}^{f}\right|} .
\end{aligned}
$$

By the same arguments, also

$$
\prod_{m \geq n}\left(1-\frac{1}{m^{2}}\right) \frac{\left|p^{f}(x)-A_{n}^{f}\right|}{\left|B_{n}^{f}-A_{n}^{f}\right|} \leq \frac{\left|p^{g}(y)-A_{n}^{g}\right|}{\left|B_{n}^{g}-A_{n}^{g}\right|} \leq \prod_{m \geq n}\left(1+\frac{1}{m^{2}}\right) \frac{\left|p^{f}(x)-A_{n}^{f}\right|}{\left|B_{n}^{f}-A_{n}^{f}\right|} .
$$

The same estimates can be derived for $x_{1}^{j} \in \operatorname{cl} T_{1}^{f}\left(x^{j}\right)=\left[p^{f}\left(x^{j}\right), q^{f}\left(x^{j}\right)\right]$ and $y_{1}^{j} \in \operatorname{cl} T_{1}^{g}\left(y^{j}\right)=\left[p^{g}\left(y^{j}\right), q^{g}\left(y^{j}\right)\right]$. Therefore

$$
\begin{aligned}
\left|y_{1}-y_{1}^{j}\right|= & \left|y_{1}-A_{n}^{g}\right|-\left|y_{1}^{j}-A_{n}^{g}\right| \\
\leq & \frac{\left|B_{n}^{g}-A_{n}^{g}\right|}{\left|B_{n}^{f}-A_{n}^{f}\right|}\left[\left|x_{1}-A_{n}^{f}\right|+\prod_{m \geq n}\left(1+\frac{1}{m^{2}}\right)-1\right] \\
& +\frac{\left|B_{n}^{g}-A_{n}^{g}\right|}{\left|B_{n}^{f}-A_{n}^{f}\right|}\left[-\left|x_{1}^{j}-A_{n}^{f}\right|+1-\prod_{m \geq n}\left(1-\frac{1}{m^{2}}\right)\right] \\
\leq & K_{2}\left[\left|x_{1}-x_{1}^{j}\right|+\prod_{m \geq n}\left(1+\frac{1}{m^{2}}\right)-\prod_{m \geq n}\left(1-\frac{1}{m^{2}}\right)\right] \rightarrow 0
\end{aligned}
$$

as $j \rightarrow \infty$ and $n \rightarrow \infty$. This proves continuity of $h$. By the same arguments also

$$
\left|y_{1}-y_{1}^{j}\right| \geq K_{1}\left[\left|x_{1}-x_{1}^{j}\right|+\prod_{m \geq n}\left(1+\frac{1}{m^{2}}\right)-\prod_{m \geq n}\left(1-\frac{1}{m^{2}}\right)\right],
$$

which yields continuity of $h^{-1}$.

The next definition is a straightforward generalization of the cutting times discussed in the Preliminaries.

Definition 3. Let $\left(I_{i}, f_{i}\right)$ be given. The cutting times $\left\{S_{i, k}\right\}$ of $\left(I_{i}, f_{i}\right)$ are given by: $S_{i, 0}=1$, and

$$
S_{i, k+1}=\min \left\{m>S_{i, k} \mid f_{i-S_{i, k}, i-m}\left(\left[c, f_{i, i-S_{i, k}}(c)\right]\right) \ni c\right\}
$$

for $i \geq 2$. 
Lemma 4. Let $\left(I_{i}, f_{i}\right)$ and $\left(J_{i}, g_{i}\right)$ be given with respective cutting times $\left\{S_{i, k}^{f}\right\}$ and $\left\{S_{i, k}^{g}\right\}$ such that for each $i$,

$$
\left\{S_{i, k}^{f}\right\}=\left\{S_{i, k}^{g}\right\}
$$

and

$$
f_{i}(c) \in L_{i-1}^{f} \quad \text { and } \quad g_{i}(c) \in L_{i-1}^{g} .
$$

Then $\left(I_{i}, f_{i}\right) \approx\left(J_{i}, g_{i}\right)$.

Proof. We need to construct the monotone bijections, $h_{i}$, as in Definition 2. Easily, $h_{1}$ exists. Assume that $h_{i-1}$ exists; we construct $h_{i}$. For $\alpha \in C_{i}^{f} \cap M_{i}^{f}$ set

$$
h_{i}(\alpha)=\left.g_{i}^{-1}\right|_{M_{i}^{g}} \circ h_{i-1} \circ f_{i}(\alpha) .
$$

As $\left.f_{i}\right|_{M_{i}^{f}}$ and $\left.g_{i}\right|_{M_{i}^{g}}$ are onto monotone maps and $h_{i-1}$ satisfies the conditions of Definition 2, it follows that $h_{i}$ satisfies the conditions of Definition 2 on $C_{i}^{f} \cap M_{i}^{f}$.

Let $\gamma_{i}^{f}=\partial L_{i}^{f} \backslash\{c\}$ and $\gamma_{i}^{g}=\partial L_{i}^{g} \backslash\{c\}$. By (14) we have $\gamma_{i}^{f}=f_{i+1}(c)$ and $\gamma_{i}^{g}=g_{i+1}(c)$. Thus, it follows from (13) that $f_{i}\left(\gamma_{i}^{f}\right) \in J_{\gamma(i)}$ and $g_{i}\left(\gamma_{i}^{g}\right) \in$ $h_{i-1}\left(J_{\gamma(i)}\right)$ for some $J_{\gamma(i)} \in \mathcal{P}_{i-1}^{f}$ and all $i$. Thus we can define $h_{i}$ on $C_{i}^{f} \cap L_{i}^{f}$ by replacing $\left.g_{i}^{-1}\right|_{M_{i}^{g}}$ in (15) with $\left.g_{i}^{-1}\right|_{L_{i}^{g}}$.

Lemma 4 can fail if we require only (13); thus having the same cutting times is not enough to guarantee combinatorial equivalence. If we replace (14) with $f_{i}(c) \in L_{i-1}^{f} \Leftrightarrow g_{i}(c) \in L_{i-1}^{g}$, then it is not necessarily true that $\left(I_{i}, f_{i}\right) \approx\left(J_{i}, g_{i}\right)$; counter-examples exist when $f_{i}(c) \in M_{i-1}^{f}$. In the proofs of Lemma 5 and Theorem 3 condition (14) holds since we defined $I \equiv\left[0, c_{1}\right]$ for $(I, f)$ with $f(0)=0$.

LeMma 5. Let $\widetilde{f}$ be a unimodal map with cutting times $\left\{\widetilde{S}_{k}\right\}$ and kneading map $\widetilde{Q}$. Assume that for a unimodal map $f$ with cutting times $\left\{S_{k}\right\}$ and kneading map $Q$ there exists an infinite (increasing) sequence $\left\{k_{i}\right\}$ such that the following properties hold:

- Let $n_{1}$ be arbitrary and set $n_{i}=n_{i-1}+S_{k_{i}}$. For all $i \geq 2$,

$$
Q\left(Q\left(k_{i}\right)+1\right)<k_{i-1} .
$$

- For all $j \geq 1$ such that $i-\widetilde{S}_{j} \geq 1$,

$$
Q\left(k_{i}+j\right)=k_{i-\widetilde{S}_{j-1}}+\widetilde{Q}(j) .
$$

Then there exists a proper subcontinuum $H$ of $(I, f)$ that is combinatorially equivalent to $(I, \tilde{f})$. 
Proof. The construction of $H$ is the same as in Proposition 2. Assumptions (i) and (ii) of Proposition 2 follow immediately from (16). Assumption (iii) of Proposition 2 follows from (17).

Again, $H=\left(\pi_{n} H, f\right)$ is homeomorphic to $\left(\pi_{n_{i}} H, f^{S_{k_{i}}}\right)$. So, let us focus on $H$ in this last form. We use Lemma 4. From the definition of $I=\left[0, c_{1}\right]$ statement (14), as discussed above, holds. Hence, we show that (13) holds. We claim: For every $r$ and every $j \geq 0$ such that $r-\widetilde{S}_{j} \geq 1$,

$$
n_{r}-S_{k_{r}+j}=n_{r-\widetilde{S}_{j}} \cdot
$$

We argue by induction, both on $r$ and on $j$. Note that $r-\widetilde{S}_{j} \geq 1 \Rightarrow 0 \leq$ $j \leq r-2$. For $r=2$, we only have to check $j=0$, and indeed $n_{2}-S_{k_{2}}=n_{1}$.

Suppose that (18) holds for all $r^{\prime}<r$ and all $j \geq 0$ such that $r^{\prime}-\widetilde{S}_{j} \geq 1$. For $j=0$, i.e. $\widetilde{S}_{j}=1$, we again have $n_{r}-S_{k_{r}}=n_{r-1}$. So let us assume that (18) holds for $r^{\prime}<r$ and also for $r^{\prime}=r$ and all $j^{\prime} \leq j$. Then

$$
\begin{aligned}
& n_{r}-S_{k_{r}+j+1}=n_{r}-S_{k_{r}+j}-S_{Q\left(k_{r}+j+1\right)} \quad(\text { by }(1)) \\
& =n_{r-\widetilde{S}_{j}}-S_{Q\left(k_{r}+j+1\right)} \quad \text { (by induction) } \\
& =n_{r-\widetilde{S}_{j}}-S_{k_{r-\tilde{S}_{j}}+\widetilde{Q}(j+1)} \quad(\text { by }(17)) \\
& =n_{r-\widetilde{S}_{j}-\widetilde{S}_{\widetilde{Q}(j+1)}}=n_{r-\widetilde{S}_{j+1}} \quad \text { (by induction and (1)), }
\end{aligned}
$$

proving the induction and the claim. Hence $\left\{S_{n, k}^{f}\right\}=\left\{S_{n, k}^{f}\right\}=\left\{S_{k}\right\}=\left\{\widetilde{S}_{k}\right\}$ for all $n$. The lemma now follows from Lemma 4 .

In the next theorem we work with unimodal maps for which the critical point is periodic. Within the tent family the set parameters for which the critical point is periodic is a dense countable set.

THEOREM 3. Let $\mathcal{F}$ be a finite or countably infinite sequence of unimodal maps, each of them having a periodic critical point. There exists $g$ such that for each $f \in \mathcal{F},(I, g)$ contains a subcontinuum $H(f)$ homeomorphic to $(I, f)$. Moreover, every proper subcontinuum $H \subset(I, g)$ that is not an arc or arc +ray continuum is homeomorphic to $(I, f)$ or $\left(\left[c_{2}, c_{1}\right], f\right)$ for some $f \in \mathcal{F}$.

Proof. For simplicity we assume that the maps $f \in \mathcal{F}$ and $g$ are tent maps. If $f$ has a periodic critical point, say of period $p$, then $f_{i, i-p}(c)=$ $f^{p}(c)=c$ for all $i>p$. Here we have written $(I, f)=\left(I_{i}, f_{i}\right)$ where $I_{i}=I$ and $f_{i}=f$ for all $i$. In particular, there exists $l$ such that $p=S_{i, l}$ for all $i>p$. Brown [9] showed that if $\tilde{f}_{i}$ are sufficiently small perturbations of $f_{i}$, then $\left(I_{i}, f_{i}\right)$ and $\left(I_{i}, \widetilde{f}_{i}\right)$ are homeomorphic. Let $S_{i, k}$ be the cutting times of $\left(I_{i}, f_{i}\right)$. We will choose these perturbations $\widetilde{f}_{i}$ such that $\widetilde{f}_{i, j}(c) \neq c$ for all $i>j \geq 1$, and $\widetilde{f}_{i, i-p}(c)$ lies on that side of $c$ that makes $p=\widetilde{S}_{i, l}$ 
a cutting time. By taking $\widetilde{f}_{i}$ sufficiently close to $f_{i}$ we can guarantee that $\widetilde{S}_{i, k}=S_{i, k}=S_{k}$ for $k \leq l$ and $\widetilde{S}_{i, l}$ is also the largest cutting time for $i$. Brown's result can be translated for our purpose into: There exists $\left\{\varepsilon_{i}\right\}$ such that if the above-described perturbations $\widetilde{f}_{i}$ satisfy $\left|\widetilde{f}_{i, i-p}(c)-c\right| \leq \varepsilon_{i}\left|I_{i-p}\right|$, then $\left(I_{i}, \widetilde{f}_{i}\right)$ is homeomorphic to $\left(I_{i}, f_{i}\right)$.

We have to construct a map $g$ such that for each map $f \in \mathcal{F}$, there exists a sequence $\left\{k_{i}\right\}_{i}$ satisfying the assumptions of Lemma 5 . Moreover, we want to keep these sequences sufficiently disjoint so that the different subcontinua will not interfere with each other.

Let $\left\{v_{s, i}\right\}_{s, i \geq 1}$ be positive integers such that $v_{s, i}<v_{t, j}$ if and only if $s+i<t+j$ or $s+i=t+j$ and $s<t$. Hence

$$
v_{1,1}<v_{1,2}<v_{2,1}<v_{1,3}<v_{2,2}<v_{3,1}<v_{1,4}<\ldots
$$

The subscript $s$ will index $\mathcal{F}$. The precise values of $v_{s, i}$ will be determined along with the construction of $g$, but at least $v_{t, j}>3 v_{s, i}$ whenever $v_{t, j}>v_{s, i}$. We define $g$ by defining its kneading map $Q$.

- Fix $s$ and let $f=f_{s}$ be the $s$ th map from $\mathcal{F}$. Consider the perturbation $\left(I_{i}, \widetilde{f}_{i}\right)$ of $(I, f)$ as above. Let $\widetilde{S}_{k}=\widetilde{S}_{i, k}$ and $\widetilde{Q}$ denote the corresponding cutting times and kneading map. For $i, j$ such that $j \geq 1$ and $i-\widetilde{S}_{j} \geq 1$, set

$$
Q\left(v_{s, i}+j\right)=v_{s, i-\widetilde{S}_{j-1}}+\widetilde{Q}(j)
$$

and if $j$ is minimal such that $i-\widetilde{S}_{j}<1$, then

$$
Q\left(v_{s, i}+j\right)=v_{s, i}-2 \text {. }
$$

- Repeat the above for all other $s$ in the index set.

- For all other integers $n, Q(n) \leq 10$ is chosen such that $Q$ as a whole becomes admissible.

Let us check that (19) and (20) allow the kneading map $Q$ to be admissible. We check the admissibility condition (3). Let $i$ be arbitrary, and let $r, j$ be such that $r \geq 0, j \geq 1$ and $i-\widetilde{S}_{r+j} \geq 1$. Write $k_{i}=v_{s, i}$. Then by (19) and using the convention that $k_{i}=v_{s, i}=0$ for $i \leq 0$, we get

$$
\left\{Q\left(k_{i}+r+j\right)\right\}_{j \geq 1}=\left\{k_{i-\widetilde{S}_{r+j-1}}+\widetilde{Q}(r+j)\right\}_{j \geq 1}
$$

and

$$
\begin{aligned}
& \left\{Q\left(Q^{2}\left(k_{i}+r\right)+j\right)\right\}_{j \geq 1}=\left\{Q\left(Q\left(k_{i-\widetilde{S}_{r-1}}+\widetilde{Q}(r)\right)+j\right)\right\}_{j \geq 1}
\end{aligned}
$$

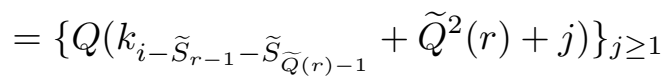

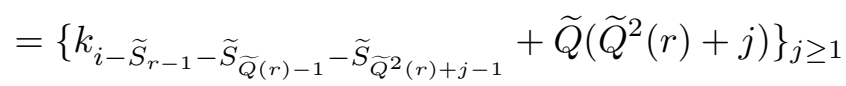

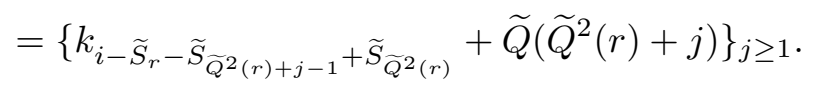


If $j=1$, then, because $\widetilde{Q}$ satisfies (3),

$Q\left(k_{i}+r+1\right)=k_{i-\widetilde{S}_{r}}+\widetilde{Q}(r+1) \geq k_{i-\widetilde{S}_{r}}+\widetilde{Q}\left(\widetilde{Q}^{2}(r)+1\right)=Q\left(Q^{2}\left(k_{i}+r\right)+1\right)$.

Now assume that $\widetilde{Q}\left(r+j^{\prime}\right)=\widetilde{Q}\left(\widetilde{Q}^{2}(r)+j^{\prime}\right)$ for all $1 \leq j^{\prime}<j$. Then using again (3) for $\widetilde{Q}$ we get

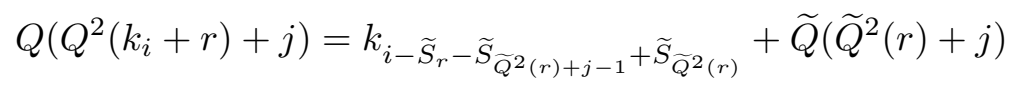

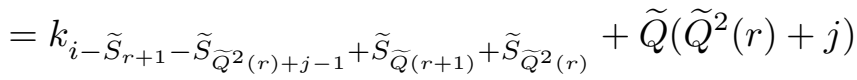

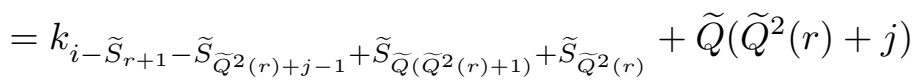

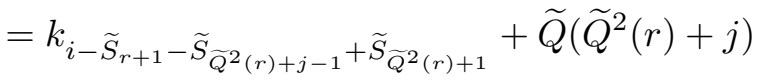

$$
\begin{aligned}
& =k_{i-\widetilde{S}_{r+j-1}}+\widetilde{Q}\left(\widetilde{Q}^{2}(r)+j\right) \\
& \leq k_{i-\widetilde{S}_{r+j-1}}+\widetilde{Q}(r+j)=Q\left(k_{i}+r+j\right) .
\end{aligned}
$$

Finally, if for fixed $r, Q\left(k_{i}+r+j\right)=Q\left(Q^{2}\left(k_{i}+r\right)+j\right)$ for all $j$ such that $i-\widetilde{S}_{r+j} \geq 1$, then we have to turn to the minimal $j$ such that $i-\widetilde{S}_{r+j}<1$. For this $j,(20)$ gives $Q\left(k_{i}+r+j\right)=k_{i}-2>Q(n)$ for all $n<k_{i}+r+j$. Therefore $Q\left(k_{i}+r+j\right)>Q\left(Q^{2}\left(k_{i}+r\right)+j\right)$. This shows that (3) holds for $Q$ as well, whence $g$ indeed exists. Note also that $Q(k)<k-1$ for all $k>1$. Therefore (see [12]) $Q$ does not belong to a renormalizable unimodal map. Hence $Q$ is indeed the kneading map of some tent map; denote this tent map by $g$ and let $J=[0, g(c)]$.

To ensure that $(J, g)$ contains the required subcontinua, define for the $s$ th map $f \in \mathcal{F}$ a sequence $\left\{n_{i}\right\}$ by $n_{1}=1$ and $n_{i}-n_{i-1}=S_{k_{i}}=S_{v_{s, i}}$. Therefore Lemma 5 yields a subcontinuum $H(f)$ of $(J, g)$ with critical projections $\left\{n_{i}\right\}$ that is combinatorially equivalent to $(I, f)$. To prove that $H(f)$ and $(I, f)$ are homeomorphic, we need to check assumption (11) of Proposition 4. For clarity, set $(J, g)=\left(J_{i}, g_{i}\right)$ where $J_{i}=J$ and $g_{i}=g$ for each $i$. In particular, $g_{n, m}=g^{n-m}: J_{n} \rightarrow J_{m}$.

Recall that $p$ is the period of the critical point of $f$ and hence $p$ is fixed; say $p \geq 3$. Take $i>p$. Assume $g_{n_{i}, n_{i-3}}(c) \in\left(a_{n_{i-3}}, b_{n_{i-3}}\right)=P_{n_{i-3}}$ for some $P_{n_{i-3}} \in \mathcal{P}_{n_{i-3}}$. Let $j$ be maximal such that $i-\widetilde{S}_{j} \geq 1$. Because $p=\widetilde{S}_{l}$ is the largest cutting time, $j=l$. Clearly, $g_{n_{i-3}, n_{i-p}}\left(P_{n_{i-3}}\right)$ $\subset P_{n_{i-p}}$ for some $P_{n_{i-p}} \in \mathcal{P}_{n_{i-p}}$. Note that the boundary points of $g_{n_{i-3}, n_{i-p}}\left(P_{n_{i-3}}\right)$ lie in $C_{n_{i-p}}$ or are points of the form $g_{n_{j}, n_{i-p}}(c)$. Because $S_{n_{i}, l}=n_{i}-n_{i-p}$ is the largest cutting time for $n_{i}, c$ is one boundary point of $g_{n_{i-3}, n_{i-p}}\left(P_{n_{i-3}}\right)$. 
By construction, $c_{S_{k_{i}+l}}=g_{n_{i}, n_{i-p}}(c)$ and by $(20)$,

$$
Q\left(v_{s, i}+l+1\right)=Q\left(k_{i}+l+1\right)=k_{i}-2 ;
$$

thus $g_{n_{i}, n_{i-p}}(c)$ becomes arbitrarily close to $c$ (recall (2)). At the same time, $g_{n_{i-3}, n_{i-p}}\left(a_{n_{i-3}}\right) \in \mathcal{C}_{n_{i-p}}$ and hence $g_{n_{i-p}, n_{r}}\left(g_{n_{i-3}, n_{i-p}}\left(a_{n_{i-3}}\right)\right)=c$ for some $1 \leq r \leq i-p$; therefore $\left|g_{n_{i-3}, n_{i-p}}\left(a_{n_{i-3}}\right)-c\right|$ has definite size. Hence, by taking $v_{s, i}=k_{i}$ sufficiently large we can make sure that

$$
\frac{\left|c-g_{n_{i}, n_{i-p}}(c)\right|}{\left|c-g_{n_{i-3}, n_{i-p}}\left(a_{n_{i-3}}^{g}\right)\right|}=\frac{\left|g_{n_{i-3}, n_{i-p}}\left(b_{n_{i-3}}^{g}\right)-g_{n_{i}, n_{i-p}}(c)\right|}{\left|g_{n_{i-3}, n_{i-p}}\left(b_{n_{i-3}}^{g}\right)-g_{n_{i-3}, n_{i-p}}\left(a_{n_{i-3}}^{g}\right)\right|}=\varepsilon<\varepsilon_{i} .
$$

Next, choose the perturbation $\tilde{f}_{i}$ of $f_{i}$ such that

$$
\left|c-\widetilde{f}_{i, i-p}(c)\right|=\varepsilon\left|c-\widetilde{f}_{i-3, i-p}\left(a_{i-3}^{\tilde{f}}\right)\right|<\varepsilon\left|I_{i-p}\right| .
$$

Here $\widetilde{f}_{i, i-3}(c) \in\left(a_{i-3}^{\widetilde{f}}, b_{i-3}^{\widetilde{f}}\right)$ is the obvious partition element of $\mathcal{P}_{i-3}^{\widetilde{f}}$. Because $\varepsilon<\varepsilon_{i}$, this perturbation stays within the scope of Brown's result. As $g_{n_{i-p}, 1}$ and $\widetilde{f}_{i-p, 1}$ are piecewise linear,

$$
\frac{\left|g_{n_{i-3}, n_{1}}\left(b_{n_{i-3}}^{g}\right)-g_{n_{i}, n_{1}}(c)\right|}{\left|g_{n_{i-3}, n_{1}}\left(b_{n_{i-3}}^{g}\right)-g_{n_{i-3}, n_{1}}\left(a_{n_{i-3}}^{g}\right)\right|}=\frac{\left|\widetilde{f}_{i-3,1}\left(b_{i-3}^{\widetilde{f}}\right)-\widetilde{f}_{i, 1}(c)\right|}{\left|\widetilde{f}_{i-3,1}\left(b_{i-3}^{\widetilde{f}}\right)-\widetilde{f}_{i-3,1}\left(a_{i-3}^{\widetilde{f}}\right)\right|} .
$$

The above proves (11).

Any number of the form $S_{v_{s, i}+j}$ with $i-\widetilde{S}_{j} \geq 1$ can be decomposed as

$$
\begin{aligned}
S_{v_{s, i}+j} & =n_{i}^{s}-n_{i-\widetilde{S}_{j}}^{s} \\
& =\left(n_{i}^{s}-n_{i-1}^{s}\right)+\left(n_{i-1}^{s}-n_{i-2}^{s}\right)+\ldots+\left(n_{i-\widetilde{S}_{j}+1}^{s}-n_{i-\widetilde{S}_{j}}^{s}\right) \\
& =S_{v_{s, i}}+S_{v_{s, i-1}}+\ldots+S_{v_{s, i-\tilde{S}_{j}+1}},
\end{aligned}
$$

and this is also the decomposition in "lowest terms". Indeed, because $v_{s, i}$ $>v_{t, j}$ implies $v_{s, i}>3 v_{t, j}$, it is not possible to decompose any of these terms into smaller terms of the form $S_{v_{t, j}}$. In general, $S_{v_{s, i}+j}$ can be written in several ways as the sum of numbers of the form $S_{v_{t, j}+r}$. But for any of the terms $t=s$. We call this set of properties the unique decomposition property.

Let us finally show that there are no other non-arc+ray subcontinua than the $H(f)$ 's in $(J, g)$. Suppose $H$ is a proper subcontinuum of $(J, g)$, and the corresponding critical projections are $\left\{m_{t}\right\}$. Because $H$ is proper, $m_{t}-m_{t-1} \rightarrow \infty$, and by applying $\widehat{g}$, we may assume that $m_{t}-m_{t-1}>S_{10}$ for all $t \geq 2$. We claim that for $t \geq 4$,

$$
m_{t}-m_{t-1}=S_{v_{\alpha(t), \beta(t)}+\gamma(t)},
$$

where $0 \leq \gamma(t)<\beta(t)$ is such that $\beta(t)-\widetilde{S}_{\gamma(t)} \geq 1$ (here $\widetilde{S}_{\gamma(t)}$ is the $\gamma(t)$ th cutting time for the map $\left.f_{\alpha(t)}\right)$. Indeed, by Lemma $3, m_{t}-m_{t-1}=S_{l_{t}}$. If $Q\left(l_{t}+1\right) \leq 10$, then (by Lemma 3 and $\left.(2)\right) m_{t-1}-m_{t-2} \leq S_{Q\left(l_{t}+1\right)} \leq S_{10}$, 
contradicting $m_{t-1}-m_{t-2}>S_{10}$. Hence there exist $\alpha(t), \beta(t)$, and $\gamma(t)$ such that $l_{t}+1=v_{\alpha(t), \beta(t)}+\gamma(t)$ with one of (19) or (20) holding; either way we have (21). This proves the claim.

Next we claim that, if $j$ is maximal such that $S_{v_{\alpha(t), \beta(t)}+\gamma(t)+j} \leq m_{t}-m_{1}$, say $S_{v_{\alpha(t), \beta(t)}+\gamma(t)+j}=m_{t}-m_{r}$, then

$$
\alpha(t)=\alpha(t-1)=\ldots=\alpha(r+1) .
$$

If $j=0$, then the statement is true by default. So assume $j \geq 1$. Take any $1 \leq j^{\prime} \leq j$ and write $S_{v_{\alpha(t), \beta(t)}+\gamma(t)+j^{\prime}}=m_{t}-m_{r^{\prime}}$ and $S_{v_{\alpha(t), \beta(t)}+\gamma(t)+j^{\prime}-1}=$ $m_{t}-m_{r^{\prime \prime}}$. Then

$$
\begin{aligned}
S_{Q\left(v_{\alpha(t), \beta(t)}+\gamma(t)+j^{\prime}\right)}= & S_{v_{\alpha(t), \beta(t)-\tilde{S}_{\gamma(t)+j^{\prime}-1}}+\widetilde{Q}\left(\gamma(t)+j^{\prime}\right)}=m_{r^{\prime \prime}}-m_{r^{\prime}} \\
= & \left(m_{r^{\prime \prime}}-m_{r^{\prime \prime}-1}\right) \\
& +\left(m_{r^{\prime \prime}-1}-m_{r^{\prime \prime}-2}\right)+\ldots+\left(m_{r^{\prime}+1}-m_{r^{\prime}}\right) .
\end{aligned}
$$

By the unique decomposition property,

$$
\alpha(t)=\alpha\left(r^{\prime \prime}\right)=\alpha\left(r^{\prime \prime}-1\right)=\ldots=\alpha\left(r^{\prime}+1\right) .
$$

This proves the second claim.

Let us call $\left\{m_{t}\right\}$ monochrome if for each $t \in \mathbb{N}, \alpha(t)$ from (21) has the same value. The claims imply that $\left\{m_{t}\right\}$ consists of monochrome blocks, and if $N=\left\{m_{a}, m_{a-1}, \ldots, m_{b}\right\}$ is such a monochrome block, say of color $s$, then for each $a \geq t \geq b$ and each $j$,

$$
S_{v_{s, t}+j} \leq m_{t}-m_{b} \quad \text { or } \quad S_{v_{s, t}+j}>m_{t}-m_{1} .
$$

If $\left\{m_{t}\right\}$ consists of finitely many finite monochrome blocks and one infinite block, say of color $s$, then the tail of $\left\{m_{t}+z\right\}$ is a subsequence of the tail of $\left\{m_{t}^{s}\right\}$ for some $z \in \mathbb{Z}$. Therefore $H \subset \widehat{g}^{-z}(H(f))$. Because $f$ is longbranched, Proposition 3 shows that either $H=g^{-z}(H(f))$, or $H$ is a point or an arc. If there are infinitely many finite monochrome blocks, then the numbers $m_{b}$ for each block form a sequence as described in Theorem 2 . In this case, $H$ is an arc+ray subcontinuum.

COROLlaRY 4. There are uncountably many non-homeomorphic unimodal inverse limit spaces.

Proof. Let $\mathcal{F}$ denote the subfamily of tent maps for which the turning point is periodic. As was pointed out in the Introduction, if $f$ is such that $c$ is periodic of period $n$ and $g$ is such that $c$ is periodic of period $m$, with $m \neq n$, then $(I, f)$ is not homeomorphic to $(I, g)$. Hence the corollary follows as the power set of $\mathcal{F}$ is an uncountable set.

We point out that one can also generate uncountably many non-homeomorphic inverse limits spaces via constructions as in Example 2. 


\section{References}

[1] J. M. Aarts and R. J. Fokkink, The classification of solenoids, Proc. Amer. Math. Soc. 111 (1991), 1161-1163.

[2] M. Barge, Horseshoe maps and inverse limits, Pacific J. Math. 121 (1986), 29-39.

[3] M. Barge, K. Brucks and B. Diamond, Self-similarity in inverse limit spaces of the tent family, Proc. Amer. Math. Soc. 124 (1996), 3563-3570.

[4] M. Barge and B. Diamond, Homeomorphisms of inverse limit spaces of onedimensional maps, Fund. Math. 146 (1995), 171-187.

[5] - - - Inverse limit spaces of infinitely renormalizable maps, Topology Appl. 83 (1998), 103-108.

[6] - - - Subcontinua of the closure of the unstable manifold at a homoclinic tangency, Ergodic Theory Dynam. Systems 19 (1999), 1-19.

[7] M. Barge and S. Holte, Nearly one-dimensional Hénon attractors and inverse limits, Nonlinearity 8 (1995), 29-42.

[8] M. Barge and J. Martin, Endpoints of inverse limit spaces and dynamics, in: Continua with the Houston Problem Book (Cincinnati, OH, 1994), Lecture Notes in Pure and Appl. Math. 170, Dekker, New York, 1995, 165-182.

[9] M. Brown, Some applications of an approximation theorem for inverse limits, Proc. Amer. Math. Soc. 11 (1960), 478-483.

[10] K. Brucks, B. Diamond, M. V. Otero-Espinar and C. Tresser, Dense orbits of critical points for the tent map, in: Contemp. Math. 117, Amer. Math. Soc., Providence, RI, 1991, 57-61.

[11] H. Bruin, Invariant measures of interval maps, Ph.D. thesis, Delft, 1994.

[12] - Combinatorics of the kneading map, Internat J. Bifur. and Chaos Appl. Sci. Engrg. 5 (1995), 1339-1349.

[13] - Planar embeddings of inverse limit spaces of unimodal maps, Topology Appl., to appear.

[14] - Inverse limit spaces of post-critically finite tent maps, preprint, 1998.

[15] W. Dębski, On topological types of the simplest indecomposable continua, Colloq. Math. 49 (1985), 203-211.

[16] F. Hofbauer, The topological entropy of the transformation $x \mapsto a x(1-x)$, Monatsh. Math. 90 (1980), 117-141.

[17] F. Hofbauer and G. Keller, Quadratic maps without asymptotic measure, Comm. Math. Phys. 127 (1990), 319-337.

[18] S. Holte, Generalized horseshoe maps and inverse limits, Pacific J. Math. 156 (1992), 297-305.

[19] W. de Melo and S. van Strien, One-Dimensional Dynamics, Springer, New York, 1993.

[20] J. Mioduszewski, Mappings of inverse limits, Colloq. Math. 10 (1963), 39-44.

[21] S. Nadler, Continuum Theory, Dekker, New York, 1992.

[22] R. C. Swanson and H. W. Volkmer, Invariants of weak equivalence in primitive matrices, preprint, 1998. 
[23] W. T. Watkins, Homeomorphic classification of certain inverse limit spaces with open bonding maps, Pacific J. Math. 103 (1982), 589-601.

[24] R. F. Williams, One-dimensional nonwandering sets, Topology 6 (1967), 473-487.

Mathematical Sciences

University of Wisconsin-Milwaukee

Milwaukee, WI 53201, U.S.A.

E-mail: kmbrucks@csd.uwm.edu
Mathematics 253-37

Caltech

Pasadena, CA 91125, U.S.A.

E-mail: bruin@cco.caltech.edu

Received 11 March 1998;

in revised form 12 February 1999 